\title{
Global translational reprogramming is a fundamental layer of immune regulation in plants
}

\author{
Guoyong Xu ${ }^{1 *}$, George H. Greene ${ }^{1 *}$, Heejin Yoo $^{1 *}$, Lijing Liu ${ }^{1}$, Jorge Marqués ${ }^{1}$, Jonathan Motley ${ }^{1}$ Xinnian Dong ${ }^{1}$
}

In the absence of specialized immune cells, the need for plants to reprogram transcription to transition from growth-related activities to defence is well understood ${ }^{1,2}$. However, little is known about translational changes that occur during immune induction. Using ribosome footprinting, here we perform global translatome profiling on Arabidopsis exposed to the microbe-associated molecular pattern elf18. We find that during this pattern-triggered immunity, translation is tightly regulated and poorly correlated with transcription. Identification of genes with altered translational efficiency leads to the discovery of novel regulators of this immune response. Further investigation of these genes shows that messenger RNA sequence features are major determinants of the observed translational efficiency changes. In the $5^{\prime}$ leader sequences of transcripts with increased translational efficiency, we find a highly enriched messenger RNA consensus sequence, R-motif, consisting of mostly purines. We show that R-motif regulates translation in response to pattern-triggered immunity induction through interaction with poly $(\mathrm{A})$-binding proteins. Therefore, this study provides not only strong evidence, but also a molecular mechanism, for global translational reprogramming during pattern-triggered immunity in plants.

Upon pathogen challenge, the first line of defence in plants involves recognition of microbe-associated molecular patterns by the pattern-recognition receptors, such as the Arabidopsis EF-Tu RECEPTOR (EFR) for the bacterial translation elongation factor EF-Tu (epitope elf18) ${ }^{3}$. Activation of pattern-recognition receptors
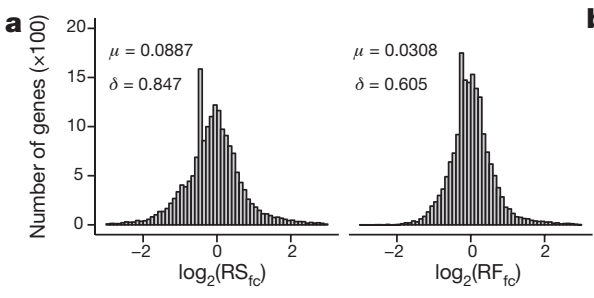

c

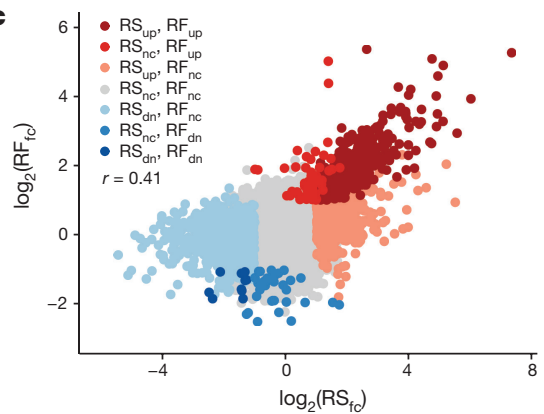

e
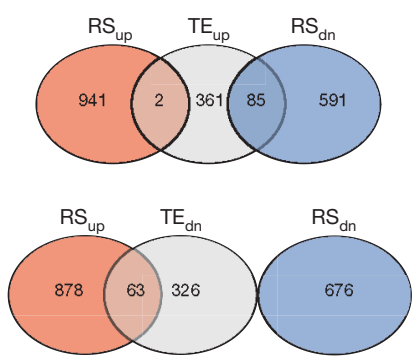

Figure 1 | Identification of novel PTI regulators on the basis of global analysis of elf18-mediated changes in translational efficiency. a, Histogram of $\log _{2}\left(\mathrm{RS}_{\mathrm{fc}}\right)$ and $\log _{2}\left(\mathrm{RF}_{\mathrm{fc}}\right)$. RS, RNA-seq; RF, ribosome footprint sequencing (or Ribo-seq); fc, fold change; $\mu$, mean; $\delta$, s.d. b, Pearson correlation coefficient $r$ between RNA-seq and Ribo-seq.
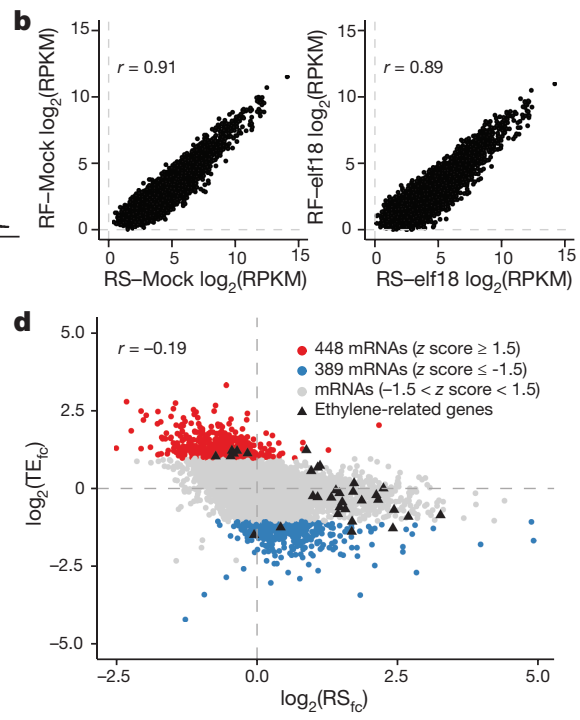

$\mathbf{f}$

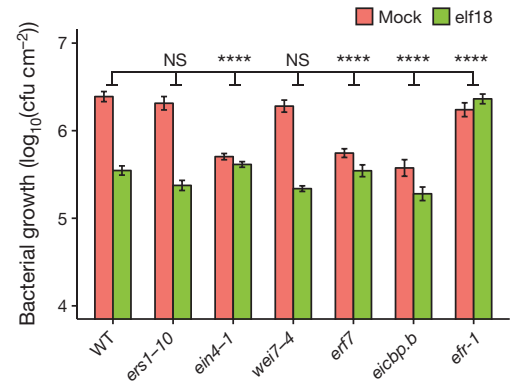

c, d, Relationships between $\mathrm{RS}_{\mathrm{fc}}$ and $\mathrm{RF}_{\mathrm{fc}}(\mathbf{c})$ and between $\mathrm{RS}_{\mathrm{fc}}$ and translational efficiency fold change $\left(\mathrm{TE}_{\mathrm{fc}}\right)(\mathbf{d})$; dn, down; nc, no change. e, Venn diagrams of $\mathrm{RS}_{\mathrm{fc}}$ and $\mathrm{TE}_{\mathrm{fc}}$. $\mathbf{f}$, The elf18-induced resistance to Psm ES4326. Mean \pm s.e.m. ( $n=12$ biological replicates from two experiments). See Extended Data Figs 4 and 5. $* * * P<0.001 ;$ NS, not significant. 
results in pattern-triggered immunity (PTI) characterized by a series of cellular changes, including MAP-kinase (MAPK) activation, ethylene biosynthesis, defence gene transcription, and enhanced resistance to pathogens ${ }^{4}$. PTI-associated transcriptional changes have been studied extensively. However, our previous report showed that in addition to transcriptional control, translation of a key immune transcription factor, TBF1, is rapidly induced during defence responses ${ }^{1}$. TBF1 translation is regulated by two upstream open reading frames (uORFs). The inhibitory effect of the uORFs on translation of the downstream major ORF (mORF) of TBF1 was rapidly alleviated upon immune induction. Similar to TBF1, translation of the Caenorhabditis elegans immune transcription factor, ZIP-2, is regulated by three $\mathrm{uORF}^{5}$, suggesting that de-repressing translation of pre-existing mRNAs of key immune transcription factors may be a common strategy for rapid response to pathogen challenge.

To monitor translational changes during immune responses, we generated an Arabidopsis transgenic line carrying the $35 \mathrm{~S}: \mathrm{uORF}_{\mathrm{TBF1}^{-}}$ LUC reporter (Extended Data Fig. 1a), whose translation, but not transcription, was induced by elf18 $1 \mathrm{~h}$ after infiltration in an $\mathrm{EFR}^{6}$ dependent manner (Extended Data Fig. 1b-e). Consistent with the reporter study, polysome profiling showed that in the absence of overall translational activity changes, the endogenous $T B F 1$ mRNA had a significant increase in association with the polysomal fractions after elf18 treatment in wild type (WT), but not in the efr-1 mutant (Extended Data Fig. 1f-i). Using conditions optimized with the $35 \mathrm{~S}: u O R \mathrm{Fs}_{\mathrm{TBF1}}-$ $L U C$ reporter, we collected leaves treated with either Mock or elf18 to generate libraries for ribosome footprint sequencing (Riboseq; also referred to as RF) and RNA sequencing (RNA-seq; also referred to as RS) (Extended Data Figs 1j, 2 and 3). Translational efficiency of mRNA was then determined by counting mRNA fragments captured by the ribosome through sequencing versus measuring the available mRNA using RNA-seq (Extended Data Fig. $4 \mathrm{a}-\mathrm{c}$ ). This strategy has previously been applied to study plant translational responses to light, hypoxia, drought, ethylene, and heat stress $^{7-11}$

We found that, upon elf18 treatment, 943 and 676 genes were transcriptionally induced $\left(\mathrm{RS}_{\mathrm{up}}\right)$ and repressed $\left(\mathrm{RS}_{\mathrm{dn}}\right)$, respectively, on the basis of differential analysis of fold change in the transcriptome $\left(\mathrm{RS}_{\mathrm{fc}}\right.$; Supplementary Table 1). Gene Ontology (GO) terms enriched for $\mathrm{RS}_{\text {up }}$ genes included defence responses (Extended Data Fig. $4 \mathrm{~d}$ ). In parallel, differential analysis of fold change in the translatome $\left(\mathrm{RF}_{\mathrm{fc}}\right)$ discovered 523 genes with increased translation $\left(\mathrm{RF}_{\mathrm{up}}\right)$ and 43 genes showing decreased translation $\left(\mathrm{RF}_{\mathrm{dn}}\right)$ (Supplementary Table 1$)$. The range of $\mathrm{RF}_{\mathrm{fc}}(0.177-40.5)$ was much narrower than that of the $\mathrm{RS}_{\mathrm{fc}}$ (0.0232-160), suggesting that translation is more tightly regulated than transcription during PTI $\left(P=3.22 \times 10^{-83}\right.$; Fig. 1a). We then calculated translational efficiency values according to a previously reported formula $^{12}$ (Extended Data Fig. 4c, e and Supplementary Table 2), using the endogenous $T B F 1$ as a positive control by counting reads to exon2 to distinguish reads from the $35 S: u O R F s_{T B F 1}-L U C$ reporter (Extended Data Fig. 4f).

In contrast to the strong correlation between levels of transcription and translation observed within the same sample (Fig. 1b), the fold changes (elf18/Mock) in transcription and translation were poorly correlated ( $r=0.41 ;$ Fig. 1c), indicating that induction of PTI involves a significant shift in global translational efficiency. Among those mRNAs
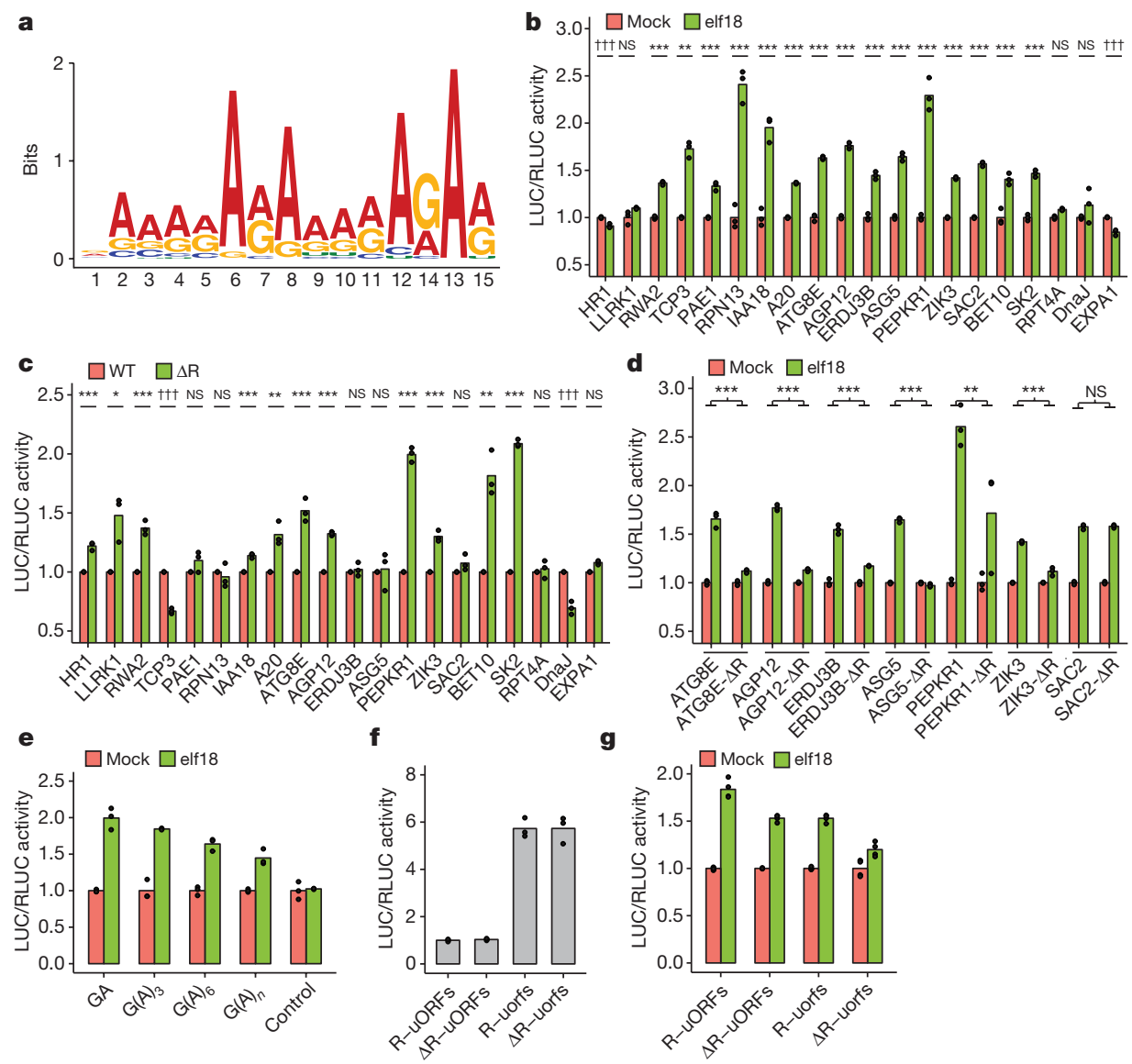

Figure 2 | Effects of R-motif on elf18-induced translation. a, R-motif consensus. b. Translational responses of $5^{\prime}$ leader sequences from 20 R-motif-containing genes. c, d, Effects of R-motif deletion mutations $(\Delta \mathrm{R})$ on basal translation $(\mathbf{c})$ and translational responsiveness to elf18 (d). e, Gain of elf18-responsiveness with $\mathrm{GA}, \mathrm{G}(\mathrm{A})_{3}, \mathrm{G}(\mathrm{A})_{6}$, and $\mathrm{G}(\mathrm{A})_{n}$ repeats. f, g, Contributions of R-motif and uORFs to TBF1 basal translation (f) and translational responsiveness to elf18 $(\mathrm{g})$. Bar with solid circles, mean with individual biological replicates $(\mathbf{b}-\mathbf{f}, n=3 ; \mathbf{g}, n=4)$. See Supplementary Table 6 and Extended Data Fig. 7. $* P<0.05, * * P<0.01, * * * P<0.001$ (all significant increase); $\uparrow+\uparrow P<0.001$ (significant decrease). 
with translational efficiency changes, 448 had increased translational efficiency fold change (GO analysis in Extended Data Fig. 4g) and 389 genes displayed decreased translational efficiency fold change $(|z| \geq 1.5)$ (Supplementary Table 1). Little correlation was found between translational efficiency changes and mRNA abundance ( $r=-0.19$; Fig. 1d, e), length, or guanine-cytosine (GC) composition (Extended Data Fig. 4h). Thus, both transcription and translational efficiency are involved in controlling protein production during PTI, and mRNA abundance is not the sole determinant of translational efficiency.

Among the genes with translational efficiency changes, we found either a known component or a homologue of a known component of nearly every step of the ethylene- and the damage-associated molecular pattern Pep-mediated PTI signalling pathways ${ }^{13}$ (Extended Data Fig. 5a and Supplementary Table 3). To demonstrate that translational efficiency measurement is an effective method of uncovering new genes involved in the elf18 signalling pathway, we tested mutants of five translational efficiency-altered genes for elf18-induced resistance against Pseudomonas syringae pathovar maculicola ES4326 (Psm ES4326). We found that ers1-10 and wei7-4 showed WT responsiveness to elf18, whereas ein4-1, erf7, and eicbp.b displayed insensitivity to elf18-induced resistance (Fig. 1f), but not MAPK3/6 activity or callose deposition (Extended Data Fig. 5b, c). Using a dual-luciferase system, we found that the $3^{\prime}$ untranslated region (UTR) of EIN4 was responsible for elf18-induced translational activity increase through the elf18 receptor, EFR (Extended Data Fig. 5d-g). The discovery of EIN4, ERF7, and EICBP.B as new PTI components on the basis of their translational efficiency changes emphasizes the utility of this approach.

To determine the potential mechanisms governing PTI-specific translation, we searched transcripts with elf18-triggered translational efficiency changes for uORFs, which have been associated with genes of different cellular functions both in plants ${ }^{14}$ and in animals ${ }^{15}$. Detailed description of the analysis and the data are shown in the Supplementary Information, Supplementary Table 4 and Extended Data Fig. 6. We also searched for consensus sequences and found one that was significantly enriched in the $5^{\prime}$ leader sequences of translational efficiency-up transcripts $\left(38.2 \%, E=1.2 \times 10^{-141}\right.$; Supplementary Table 5) compared with all Arabidopsis transcripts (17.7\%) and all translated transcripts in this translatome analysis (24.3\%; Supplementary Table 5). Since this element contains almost exclusively purines (Fig. 2a), we named it 'R-motif' (International Union of Pure and Applied Chemistry code).

To examine the effect of R-motif on elf18-induced translation, we tested $5^{\prime}$ leader sequences of 20 R-motif-containing translational efficiency-up genes using the dual-luciferase system (Extended Data Fig. 5d). Consistent with their known importance in controlling translation ${ }^{16}$, the different $5^{\prime}$ leader sequences showed distinct basal translational activities (Extended Data Fig. 7a), and elf18-mediated translational activity increase was confirmed in 15 of them (Fig. 2b). We then generated R-motif deletion and multi-base-pair substitution mutant reporters and found that 11 of them showed increased translational activity while only two displayed decreased activity compared with their corresponding WT controls (Fig. 2c and Extended Data Fig. $7 b-f)$. These results suggest a predominantly negative role for R-motif in basal translational activity. We subsequently found six R-motif deletion mutants to have abolished or decreased responsiveness to elf18 induction compared with the controls (Fig. 2d and Extended Data Fig. $7 g, h$ ), indicating that releasing R-motif-mediated repression may be an activation mechanism for these genes during PTI. To demonstrate that R-motif is sufficient for responsiveness to elf18, we tested repeats of $\mathrm{GA}, \mathrm{G}(\mathrm{A})_{3}, \mathrm{G}(\mathrm{A})_{6}$, and mixed $\mathrm{G}(\mathrm{A})_{n}$, which are core sequence patterns found in R-motifs of endogenous genes, and discovered that translation of resulting reporters indeed became responsive to elf18 induction (Fig. 2e and Extended Data Fig. 7i). However, R-motif may have a more complex role in those genes in which deleting R-motif did not affect elf18-mediated translation (Extended Data Fig. 7h). Other mRNA sequence features in these transcripts may influence R-motif activity.
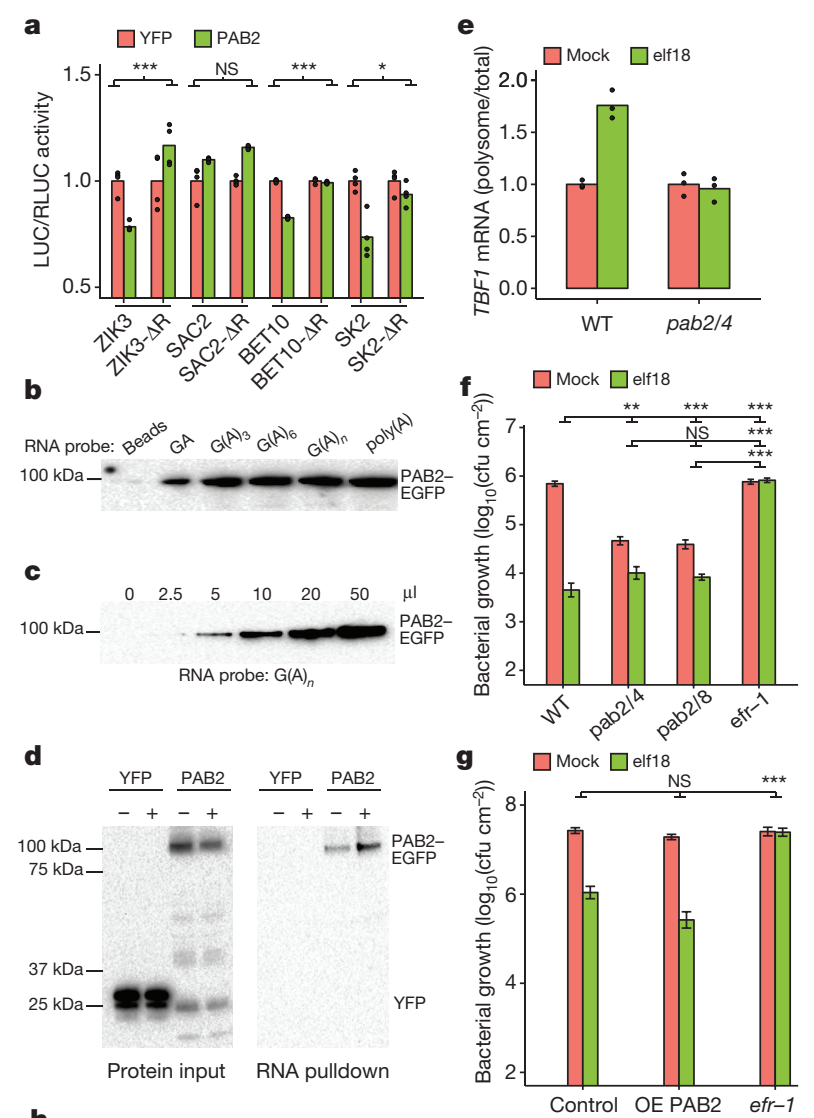

h
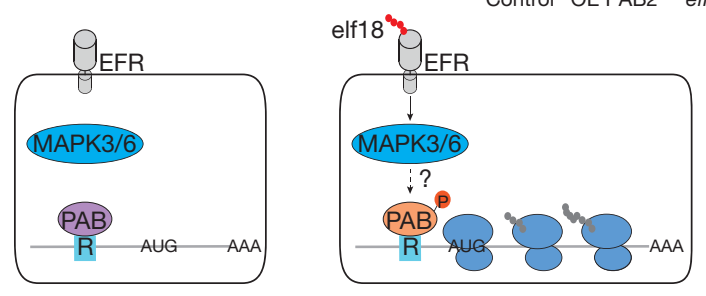

Figure 3 | R-motif controls translation through PAB. a, Effects of PAB2 on translation of R-motif-containing genes. $\mathbf{b}$-d, R-motif-binding by in vitro $(\mathbf{b}, \mathbf{c})$ and in vivo synthesized PAB2 (d); - or +, Mock or elf18-treated plants. e, TBF1 translation in pab2/4 plant. f, g, The elf18-induced resistance to Psm ES4326 in pab2/4 and pab2/8 plants (f), and in primary transformants overexpressing $\mathrm{PAB} 2(\mathrm{OE}-\mathrm{PAB} 2)(\mathrm{g})$, mean \pm s.e.m. h. Working model. Bar with solid circles, mean with individual biological replicates. See Extended Data Fig. 8 and Source Data for sample size $(n)$ and Supplementary Text for gel source data. $* P<0.05$, ** $P<0.01$, *** $P<0.001$.

The relationship between R-motif and uORFs was then studied in the $T B F 1$ transcript, which contains both features (Extended Data Fig. 1a). Translational activity assessment using the dual-luciferase system showed that deletion of R-motif had no significant effect on basal translation of the reporter, in contrast to the $\mathrm{uORFs}_{\mathrm{TBF} 1}$ mutant (Fig. $2 \mathrm{f}$ and Extended Data Fig. 7j). However, both R-motif and uORFs mutant reporters showed compromised responses to elf18 in transient expression analysis as well as in transgenic plants (Fig. $2 \mathrm{~g}$ and Extended Data Fig. 7k, 1). The effects seemed to be additive, suggesting that R-motif and uORFs control translation through distinct mechanisms.

We hypothesized that the mechanism by which R-motif affects translation is probably through association with poly $(\mathrm{A})$-binding proteins (PABs) because these proteins have been shown to bind not only to poly(A) tails of transcripts to enhance translation but also to A-rich sequences located in their own $5^{\prime}$ leader sequences to inhibit translation ${ }^{17}$. To test our hypothesis, we examined the role of class II $\mathrm{PABs}$ (that is, $\mathrm{PAB} 2, \mathrm{PAB} 4$, and $\mathrm{PAB} 8)^{18}$ by co-expressing $\mathrm{PAB} 2$ with three R-motif-dependent genes, ZIK3, BET10, and SK2, and one 
R-motif-independent gene, $S A C 2$. We found that all three R-motifdependent genes, but not the control, had lower translational activity when PAB2 was co-expressed, and that this inhibition could be overcome by deleting the R-motif (Fig. 3a and Extended Data Fig. 8a). This PAB2 effect is probably through a direct physical interaction with R-motif, because in an in vitro binding assay, PAB2 displayed comparable affinities to $\mathrm{G}(\mathrm{A})_{3}, \mathrm{G}(\mathrm{A})_{6}$, and $\mathrm{G}(\mathrm{A})_{n}$ repeats as to poly $(\mathrm{A})$ (Fig. 3b, c). Moreover, plant-synthesized PAB2 could be pulled down using a $\mathrm{G}(\mathrm{A})_{n}$ RNA probe (Fig. 3d). Surprisingly, PAB2 from elf18induced plants seemed to bind the probe more tightly than the Mocktreated control, suggesting elf18-triggered de-repression was unlikely owing to dissociation of PAB2. PAB2 is known to switch its activity through phosphorylation ${ }^{19}$, which might have occurred upon elf18 treatment.

We next examined the phenotypes of the pab2/4 and pab2/8 double mutants ${ }^{18}$. We found that the elf18-triggered increase in polysomeassociation of the endogenous TBF1 mRNA was compromised in the pab2/4 double mutant (Fig. 3e). The inhibitory effect of elf18 on plant growth was also diminished in the double mutants (Extended Data Fig. 8b). Compared with WT, the double mutants had significantly elevated basal resistance to Psm ES4326, but reduced resistance to the pathogen after elf18 treatment (Fig. 3f). This insensitivity to elf18 was rescued by transformation of PAB2 (Fig. $3 \mathrm{~g}$ ). These data support our hypothesis that PABs play a negative role in basal translation, but a positive role in elf18-induced translation. Whether the activities of PABs are regulated by components of the known PTI signalling pathway, such as MAPK3/6, remains to be tested. Detection of MAPK3/6 activity in the pab2/4 and pab2/8 mutants (Extended Data Fig. $8 \mathrm{c}$ ) suggests that PABs could function downstream of MAPK3/6, possibly as substrates (Fig. 3h).

Immune-related translation has not been well studied in any organism. In this study, we found that the inhibitory effects of uORFs and R-motif on PTI-associated genes are rapidly alleviated upon immune induction in Arabidopsis. In yeast, uORF inhibition on GCN4 translation is removed during amino-acid starvation through GCN2 mediated phosphorylation of the translation initiation factor eIF $2 \alpha^{20}$. Surprisingly, GCN2-mediated eIF2 $\alpha$ phosphorylation is not required for elf18-induced TBF1 translation or resistance (Extended Data Fig. 9), suggesting an alternative mechanism in immune-induced translational reprogramming in plants. Further investigation will also be required to dissect the regulatory mechanisms of R-motifs and understand the roles of PABs in different translation mechanisms ${ }^{21}$. Intriguingly, R-motif is also prevalent in mRNAs from other organisms (Supplementary Table 5), including the human p53 mRNA, suggesting a conserved regulatory mechanism may be shared across species.

Online Content Methods, along with any additional Extended Data display items and Source Data, are available in the online version of the paper; references unique to these sections appear only in the online paper.

Received 8 September 2016; accepted 19 April 2017. Published online 17 May 2017.

1. Pajerowska-Mukhtar, K. M. et al. The HSF-like transcription factor TBF1 is a major molecular switch for plant growth-to-defense transition. Curr. Biol. 22, 103-112 (2012).

2. Huot, B., Yao, J., Montgomery, B. L. \& He, S. Y. Growth-defense tradeoffs in plants: a balancing act to optimize fitness. Mol. Plant 7, 1267-1287 (2014).

3. Couto, D. \& Zipfel, C. Regulation of pattern recognition receptor signalling in plants. Nature Rev. Immunol. 16, 537-552 (2016).
4. Wu, S., Shan, L. \& He, P. Microbial signature-triggered plant defense responses and early signaling mechanisms. Plant Sci. 228, 118-126 (2014).

5. Dunbar, T. L., Yan, Z., Balla, K. M., Smelkinson, M. G. \& Troemel, E. R. C. elegans detects pathogen-induced translational inhibition to activate immune signaling. Cell Host Microbe 11, 375-386 (2012).

6. Zipfel, C. et al. Perception of the bacterial PAMP EF-Tu by the receptor EFR restricts Agrobacterium-mediated transformation. Cell 125, 749-760 (2006)

7. Lei, L. et al. Ribosome profiling reveals dynamic translational landscape in maize seedlings under drought stress. Plant J. 84, 1206-1218 (2015).

8. Merchante, C. et al. Gene-specific translation regulation mediated by the hormone-signaling molecule EIN2. Cell 163, 684-697 (2015).

9. Liu, M. J. et al. Translational landscape of photomorphogenic Arabidopsis. Plant Cell 25, 3699-3710 (2013).

10. Juntawong, P., Girke, T., Bazin, J. \& Bailey-Serres, J. Translational dynamics revealed by genome-wide profiling of ribosome footprints in Arabidopsis. Proc. Natl Acad. Sci. USA 111, E203-E212 (2014).

11. Lukoszek, R., Feist, P. \& Ignatova, Z. Insights into the adaptive response of Arabidopsis thaliana to prolonged thermal stress by ribosomal profiling and RNA-seq. BMC Plant Biol. 16, 221 (2016).

12. Ingolia, N. T., Ghaemmaghami, S., Newman, J. R. S. \& Weissman, J. S. Genome-wide analysis in vivo of translation with nucleotide resolution using ribosome profiling. Science 324, 218-223 (2009).

13. Zipfel, C. Combined roles of ethylene and endogenous peptides in regulating plant immunity and growth. Proc. Natl Acad. Sci. USA 110, 5748-5749 (2013)

14. von Arnim, A. G., Jia, Q. \& Vaughn, J. N. Regulation of plant translation by upstream open reading frames. Plant Sci. 214, 1-12 (2014).

15. Barbosa, C., Peixeiro, I. \& Romão, L. Gene expression regulation by upstream open reading frames and human disease. PLoS Genet. 9, e1003529 (2013)

16. Hinnebusch, A. G., Ivanov, I. P. \& Sonenberg, N. Translational control by 5'-untranslated regions of eukaryotic mRNAs. Science 352, 1413-1416 (2016).

17. Patel, G. P., Ma, S. \& Bag, J. The autoregulatory translational control element of poly(A)-binding protein mRNA forms a heteromeric ribonucleoprotein complex. Nucleic Acids Res. 33, 7074-7089 (2005).

18. Dufresne, P. J., Ubalijoro, E., Fortin, M. G. \& Laliberté, J. F. Arabidopsis thaliana class II poly(A)-binding proteins are required for efficient multiplication of turnip mosaic virus. J. Gen. Virol. 89, 2339-2348 (2008)

19. Gallie, D. R. The role of the poly $(A)$ binding protein in the assembly of the Cap-binding complex during translation initiation in plants. Translation 2, e959378 (2014).

20. Hinnebusch, A. G. Translational regulation of GCN4 and the general amino acid control of yeast. Annu. Rev. Microbiol. 59, 407-450 (2005).

21. Gilbert, W. V., Zhou, K., Butler, T. K. \& Doudna, J. A. Cap-independent translation is required for starvation-induced differentiation in yeast. Science $\mathbf{3 1 7}$, 1224-1227 (2007).

Supplementary Information is available in the online version of the paper.

Acknowledgements This study was supported by grants from National Institutes of Health 5R01 GM069594-11 and the Howard Hughes Medical Institute and the Gordon and Betty Moore Foundation (through grant GBMF3032) to X. Dong. We thank J. M. Alonso for ein4-1, wei7-4, and ers 1-10 seeds; T. Girke, M. Hummel, and J. Bailey-Serres for providing the Ribo-Seq workflow package for data analyses; W. Wang, P. Y. Hsu, and P. N. Benfey for discussing the protocol; R. Zavaliev for the callose staining method; and the Arabidopsis Information Resource for gcn2, erf7, eicbp.b, pab2/4, and pab2/8 seeds. We thank P. Zwack and S. Zebell for comments on the manuscript.

Author Contributions G.X. and X.D. designed the research. G.X., H.Y. and J. Marqués optimized the footprinting protocol. H.Y. and G.X. performed ethylene-related and polysome profiling experiments. L.L. and G.X. generated the reporter lines. G.X. and J. Motley performed elF2 $\alpha$ phosphorylation assay. G.X. performed the rest of the experiments. G.G. and G.X. performed the bioinformatic analyses and prepared the figures. G.X. and X.D. wrote the manuscript with input from all authors.

Author Information Reprints and permissions information is available at www.nature.com/reprints. The authors declare competing financial interests: details are available in the online version of the paper. Readers are welcome to comment on the online version of the paper. Publisher's note: Springer Nature remains neutral with regard to jurisdictional claims in published maps and institutional affiliations. Correspondence and requests for materials should be addressed to X.D. (xdong@duke.edu). 


\section{METHODS}

No statistical methods were used to predetermine sample size. The experiments were not randomized. The investigators were not blinded to allocation during experiments and outcome assessment.

Plasmids. To construct the $35 \mathrm{~S}: u O R F s_{T B F I}-L U C$ reporter, the $35 \mathrm{~S}$ promoter and the TBF1 exon1 (including the R-motif, $u O R F 1-u O R F 2$, and the coding sequence of the first 73 amino acids of TBF1) were amplified from p35S:uORF1-uORF2 GUS $^{1}$ using Reporter-F/R primers, and ligated into pGWB235 (ref. 22) via gateway recombination. The $35 \mathrm{~S}: c c d B$ cassette- $L U C-N O S$ construct was generated by fusing PCR fragments of the $35 \mathrm{~S}$ promoter from pMDC140 (ref. 23), the ccdB cassette, and the NOS terminator from pRNAi-LIC ${ }^{24}$ and LUC from pGWB235 (ref. 22). The 35S:ccdB cassette-LUC-NOS was then inserted into pCAMBIA1300 via PstI and EcoRI and designated as pGX301 for cloning $5^{\prime}$ leader sequences through replacement of the ApaI-flanked ccdB cassette ${ }^{24}$. Similarly, the 35S:RLUC-HArbs terminator construct was made through fusion of PCR fragments of $35 \mathrm{~S}$ from pMDC140 (ref. 23), RLUC from pmirGLO (Promega, E1330), and rbs terminator from pCRG3301 (ref. 25). The 35S:RLUC-HA-rbs fragment flanked with EcoRI was inserted into pTZ-57rt (Thermo Fisher, K1213) via TA cloning to generate pGX125. The $5^{\prime}$ leader sequences were amplified from the Arabidopsis (Col-0) genomic DNA or synthesized by Bio Basics (New York, USA) and inserted into pGX301 followed by transferring 35S:RLUC-HA-rbs from pGX125 via EcoRI. EFR, PAB2, PAB4, and PAB8 were amplified from U21686, C104970, U10212, and U15101 (from the Arabidopsis Biological Resource Center), respectively, and fused with the amino $(\mathrm{N})$ terminus of enhanced green fluorescent protein (EGFP) by PCR. Fusion fragments were then inserted between the $35 \mathrm{~S}$ promoter and the rbs terminator to generate 35S:EFR-EGFP (pGX664), 35S:EFR (pGX665), and 35S:PAB2-EGFP (pGX694). Information on all plasmids and primers in this study can be found in Supplementary Table 6.

Plant growth, transformation, and treatment. Plants were grown on soil (Metro Mix 360 ) at $22^{\circ} \mathrm{C}$ under $12 / 12$-h light/dark cycles with $55 \%$ relative humidity. Mutants efr-1 (ref. 6), ers1-10 (a weak gain-of-function mutant; ERS, ethylene receptor-related gene family member) ${ }^{26}$, ein $4-1$ (a gain-of-function mutant; EIN4, ethylene receptor-related gene family member ${ }^{27}$, wei7-4 (a loss-of-function mutant; WEI7, involved in ethylene-mediated auxin increase) ${ }^{28}$, eicbp.b (camta 1-3 SALK_108806; EICBP.B, an ethylene-induced calmodulin-binding protein $)^{29}$, and pab2/4 (ref. 18) and pab2/8 (ref. 18) were previously described; erf7 (SALK_205018; ERF7, a homologue of the ethylene responsive transcription factor gene ERF1) and gcn2 (GABI_862B02) were from the Arabidopsis Biological Resource Center. Transgenic plants were generated using the floral dip method ${ }^{30}$

Ribo-seq library construction. Leaves from $\sim 243$-week-old plants (two leaves per plant; $\sim 1.0 \mathrm{~g}$ ) were collected. Tissue was fast frozen and ground in liquid nitrogen. Five millilitres of cold polysome extraction buffer (PEB; $200 \mathrm{mM}$ Tris pH 9.0, $200 \mathrm{mM} \mathrm{KCl}, 35 \mathrm{mM} \mathrm{MgCl}_{2}, 25 \mathrm{mM}$ EGTA, 5 mM DTT, $1 \mathrm{mM}$ phenylmethanesulfonylfluoride (PMSF), $50 \mu \mathrm{g} \mathrm{ml}^{-1}$ cycloheximide, $50 \mu \mathrm{g} \mathrm{ml}^{-1}$ chloramphenicol, 1\% (v/v) Brij-35, 1\% (v/v) Igepal CA630, 1\% (v/v) Tween 20, 1\% (v/v) Triton X-100, $1 \%$ sodium deoxycholate (DOC), $1 \%(\mathrm{v} / \mathrm{v})$ polyoxyethylene 10 tridecyl ether (PTE)) was added. After thawing on ice for $10 \mathrm{~min}$, lysate was centrifuged at $4^{\circ} \mathrm{C} / 16,000 \mathrm{~g}$ for $2 \mathrm{~min}$. Supernatant was transferred to $40 \mu \mathrm{m}$ filter falcon tube and centrifuged at $4^{\circ} \mathrm{C} / 7,000 \mathrm{~g}$ for $1 \mathrm{~min}$. Supernatant was then transferred into a 2 - $\mathrm{ml}$ tube and centrifuged at $4^{\circ} \mathrm{C} / 16,000 \mathrm{~g}$ for $15 \mathrm{~min}$ and this step was repeated once. Lysate $(0.25 \mathrm{ml})$ was saved for total RNA extraction for making the RNAseq library. Another $1 \mathrm{ml}$ of lysate was layered on top of $0.9 \mathrm{ml}$ sucrose cushion ( $400 \mathrm{mM}$ Tris. $\mathrm{HCl} \mathrm{pH}$ 9.0, $200 \mathrm{mM} \mathrm{KCl}, 35 \mathrm{mM} \mathrm{MgCl}_{2}, 1.75 \mathrm{M}$ sucrose, $5 \mathrm{mM}$ DTT, $50 \mu \mathrm{g} \mathrm{ml}^{-1}$ chloramphenicol, $50 \mu \mathrm{g} \mathrm{ml}^{-1}$ cycloheximide) in an ultracentrifuge tube (349623, Beckman). The samples were then centrifuged at $4^{\circ} \mathrm{C} / 70,000$ r.p.m. for $4 \mathrm{~h}$ in a TLA100.1 rotor. The pellet was washed twice with cold water, resuspended in $300 \mu \mathrm{l}$ RNase I digestion buffer ( $20 \mathrm{mM}$ Tris. $\mathrm{HCl} \mathrm{pH} \mathrm{7.4,} 140 \mathrm{mM} \mathrm{KCl}, 35 \mathrm{mM}$ $\mathrm{MgCl}_{2}, 50 \mu \mathrm{g} \mathrm{ml}^{-1}$ cycloheximide, $50 \mu \mathrm{g} \mathrm{ml}^{-1}$ chloramphenicol) ${ }^{10}$ and then transferred to a new tube for brief centrifugation. The supernatant was then transferred to another new tube where $10 \mu \mathrm{l}$ RNase I $\left(100 \mathrm{U}_{\mu l}^{-1}\right)$ was added before $60 \mathrm{~min}$

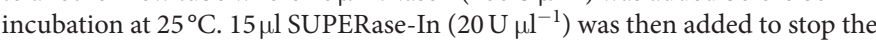
reaction. The subsequent steps including ribosome recovery, footprint fragment purification, PNK treatment, and linker ligation were performed as previously reported ${ }^{31}$. Two and a half microlitres of $5^{\prime}$ deadenylase (NEB) were then added to the ligation system and incubated at $30^{\circ} \mathrm{C}$ for $1 \mathrm{~h}$. Two and a half microlitres of $\operatorname{Rec}_{\mathrm{f}}$ exonuclease (NEB) was subsequently added for $1 \mathrm{~h}$ incubation at $37^{\circ} \mathrm{C}$. The enzymes were inactivated at $70^{\circ} \mathrm{C}$ for $20 \mathrm{~min}$ and $10 \mu \mathrm{l}$ of the samples were taken as template for reverse transcription (Extended Data Fig. 2). The rest of the steps for the library construction were performed as in the reported protocol ${ }^{31}$, with the exception of using biotinylated oligos, rRNA1 and rRNA2, for Arabidopsis according to another reported method $^{10}$.

RNA-seq library construction. TRIzol LS $(0.75 \mathrm{ml}$; Ambion) was added to the $0.25 \mathrm{ml}$ lysate saved from the Ribo-seq library construction, from which total
RNA was extracted, quantified, and qualified using Nanodrop (Thermo Fisher Scientific). Total RNA (50-75 $\mu$ g) was used for mRNA purification with Dynabeads Oligo (dT) ${ }_{25}$ (Invitrogen). Twenty microlitres of the purified poly (A) mRNA was mixed with $20 \mu \mathrm{l} 2 \times$ fragmentation buffer $\left(2 \mathrm{mM}\right.$ EDTA, $10 \mathrm{mM} \mathrm{Na}_{2} \mathrm{CO}_{3}, 90 \mathrm{mM}$ $\mathrm{NaHCO}_{3}$ ) and incubated for $40 \mathrm{~min}$ at $95^{\circ} \mathrm{C}$ before cooling on ice. Five hundred microlitres of cold water, $1.5 \mu \mathrm{l}$ of GlycoBlue, and $60 \mu \mathrm{l}$ of cold $3 \mathrm{M}$ sodium acetate were then added to the samples and mixed. Subsequently, $600 \mu \mathrm{l}$ isopropanol was added before precipitation at $-80^{\circ} \mathrm{C}$ for at least $30 \mathrm{~min}$. Samples were then centrifuged at $4^{\circ} \mathrm{C} / 15,000 \mathrm{~g}$ for $30 \mathrm{~min}$ to remove all liquid and air dried for $10 \mathrm{~min}$ before resuspension in $5 \mu \mathrm{l}$ of $10 \mathrm{mM}$ Tris $\mathrm{pH}$ 8. The rest of the steps were the same as Ribo-seq library preparation with quality control data shown in Extended Data Fig. 3.

LUC reporter assay and dual-luciferase assay. To record the $35 S: u O R F_{T_{B B F}-L U C}$ reporter activity, 3-week-old Arabidopsis plants were sprayed with $1 \mathrm{mM}$ luciferin $12 \mathrm{~h}$ before infiltration with either $10 \mu \mathrm{M}$ elf 18 (synthesized by GenScript) or $10 \mathrm{mM} \mathrm{MgCl}_{2}$ as Mock. Luciferase activity was recorded in a CCD (charge-coupled device) camera-equipped box (Lightshade Company) with each exposure time of $20 \mathrm{~min}$. For the dual-luciferase assay, Nicotiana benthamiana plants were grown at $22^{\circ} \mathrm{C}$ under $12 / 12$-h light/dark cycles. Dual-luciferase constructs were transformed into the Agrobacterium strain GV3101, which was cultured overnight at $28^{\circ} \mathrm{C}$ in Luria-Bertani broth supplied with kanamycin $\left(50 \mathrm{mg} \mathrm{l}^{-1}\right)$, gentamycin $\left(50 \mathrm{mg} \mathrm{l}^{-1}\right)$, and rifampicin $\left(25 \mathrm{mg} \mathrm{l}^{-1}\right)$. Cells were then spun down at $2,600 \mathrm{~g}$ for $5 \mathrm{~min}$, resuspended in infiltration buffer $(10 \mathrm{mM} 2$-( $N$-morpholino) ethanesulfonic acid (MES), $10 \mathrm{mM} \mathrm{MgCl}_{2}, 200 \mu \mathrm{M}$ acetosyringone), adjusted to an opitcal density at $600 \mathrm{~nm}\left(\mathrm{OD}_{600 \mathrm{~nm}}\right)=0.1$, and incubated at room temperature for an additional $4 \mathrm{~h}$ before infiltration using $1 \mathrm{ml}$ needleless syringes. For elf 18 induction, $10 \mathrm{mM} \mathrm{MgCl}_{2}$ (Mock) solution or $10 \mu \mathrm{M}$ elf18 were infiltrated $20 \mathrm{~h}$ after the dualluciferase construct and EFR-EGFP had been co-infiltrated at the ratio of 1:1, and samples were collected $2 \mathrm{~h}$ after treatment. For the PAB2-EGFP co-expression assay, Agrobacterium containing a dual-luciferase construct was mixed with Agrobacterium containing the PAB2-EGFP construct at a ratio of 1:5. Leaf discs were collected, ground in liquid nitrogen, and lysed with the PLB buffer (Promega, E1910). Lysate was spun down at $15,000 \mathrm{~g}$ for $1 \mathrm{~min}$, from which $10 \mu \mathrm{l}$ was used for measuring LUC and RLUC activity using a Victor3 plate reader (PerkinElmer). At $25^{\circ} \mathrm{C}$, substrates for LUC and RLUC were added using the automatic injector and after $3 \mathrm{~s}$ shaking and $3 \mathrm{~s}$ delay, the signals were captured for $3 \mathrm{~s}$ and recorded as counts per second.

elf18-induced growth inhibition and resistance to Psm ES4326. For elf18induced growth inhibition assay, seeds were sterilized in a $2 \%$ PPM solution (Plant Cell Technology) at $4{ }^{\circ} \mathrm{C}$ for 3 days and sown on MS media (1/2 MS basal salts, $1 \%$ sucrose, and $0.8 \%$ agar) with or without $100 \mathrm{nM}$ elf18. Ten-day-old seedlings were weighed with ten seedlings per sample. For elf18-induced resistance to Psm ES4326, $1 \mu \mathrm{M}$ elf18 or Mock (10 $\mathrm{mM} \mathrm{MgCl}_{2}$ ) was infiltrated into 3-week-old soil-grown plants 1 day before Psm ES4326 $\left(\mathrm{OD}_{600 \mathrm{~nm}}=0.001\right)$ infection of the same leaf. Bacterial growth was scored 3 days after infection. For elf18-induced resistance to Psm ES4326 in primary transformants overexpressing PAB2 in the pab2/8 mutant (OE-PAB2), transgenic plants expressing yellow fluorescent protein (YFP) in the WT background were used as control, and both control and OE-PAB2 were selected for basta-resistance and further confirmed by PCR.

elf18-induced MAPK activation and callose deposition. For MAPK activation 12-day-old seedlings grown on MS media were flooded with $1 \mu \mathrm{M}$ elf18 solution and 25 seedlings were collected at the indicated time points. Protein was extracted with co-IP buffer (50 mM Tris, pH 7.5, $150 \mathrm{mM} \mathrm{NaCl}, 0.1 \%$ (v/v) Triton X-100, $0.2 \%(\mathrm{v} / \mathrm{v})$ Nonidet P-40, protease inhibitor cocktail (Roche), phos-stop phosphatase inhibitor cocktail (Roche)). Antibody information and conditions can be found in Supplementary Table 6. For callose deposition, 3-week-old soil-grown plants were infiltrated with $1 \mu \mathrm{M}$ elf18. After $20 \mathrm{~h}$ of incubation, leaves were collected, decolorized in $100 \%$ ethanol with gentle shaking for $4 \mathrm{~h}$, and rehydrated in water for $30 \mathrm{~min}$ before stained in $0.01 \%(\mathrm{w} / \mathrm{v})$ aniline blue in $0.01 \mathrm{M} \mathrm{K}_{3} \mathrm{PO}_{4} \mathrm{pH}$ 12 covered with aluminium foil for $24 \mathrm{~h}$ with gentle shaking. Callose deposition was observed with a Zeiss-510 inverted confocal microscope using a $405 \mathrm{~nm}$ laser for excitation and $420-480 \mathrm{~nm}$ filter for emission.

RNA pull-down of in vitro and in vivo synthesized PAB proteins. PAB2-EGFP was amplified from pGX694. GA, $G(A)_{3}$, and $G(A)_{6}$ were synthesized using Bio Basics (New York, USA) while poly(A) and G(A) $)_{n}$ were synthesized by IDT (https://www.idtdna.com/site). The sequences used for in vitro biotin-RNA synthesis can be found in Supplementary Table 6. In vitro transcription and translation were performed using the wheat germ translation system according to the manufacturer's instructions (BioSieg, Japan). To make biotin-labelled RNA probes, $2 \mu \mathrm{l}$ of $10 \mathrm{mM}$ biotin-16-UTP (11388908910, Roche) was added into the transcription system. DNase I was then used to remove the DNA template. Biotin-labelled RNA $(0.2 \mathrm{nmol})$ was conjugated to $50 \mu \mathrm{l}$ streptavidin magnetic beads $(65001$ Thermo Fisher) according to the manufacturer's instructions. In vitro synthesized 
PAB2-EGFP was incubated with biotin-labelled RNA in the glycerol-co-IP buffer (50 mM Tris, pH 7.5, $150 \mathrm{mM} \mathrm{NaCl}, 2.5 \mathrm{mM}$ EDTA, 10\% (v/v) glycerol, $1 \mathrm{mM}$ PMSF, $20 \mathrm{U} \mathrm{ml}^{-1}$ Super-In RNase inhibitor, protease inhibitor cocktail (Roche)). To perform in vivo pull-down experiment, PAB2-EGFP was co-expressed with the elf18 receptor EFR (pGX665) for $40 \mathrm{~h}$ in $N$. benthamiana, which was then treated with Mock or elf18 for $2 \mathrm{~h}$. Protein was extracted with glycerol-co-IP buffer and used in the pull-down assay at $4^{\circ} \mathrm{C}$ for $4 \mathrm{~h}$. YFP was expressed as a control. Antibody information and assay conditions can be found in Supplementary Table 6.

Polysome profiling. Arabidopsis tissue $(0.6 \mathrm{~g})$ was ground in liquid nitrogen with $2 \mathrm{ml}$ cold PEB buffer. One millilitre of crude lysate was loaded to $10.8 \mathrm{ml} 15-60 \%$ sucrose gradient and centrifuged at $4{ }^{\circ} \mathrm{C}$ for $10 \mathrm{~h}$ (35,000 r.p.m., SW 41 Ti rotor). $A_{254 \mathrm{~nm}}$ absorbance recording and fractionation were performed as described previously $^{32}$. Polysomal RNA was isolated by pelleting polysomes, and polysomal/ total mRNA ratio was calculated as described previously ${ }^{8}$.

Real-time reverse-transcription polymerase chain reaction. About $50 \mathrm{mg}$ of leaf tissue was used for total RNA extraction using TRIzol following the manufacturer's instructions (Ambion). After DNase I (Ambion) treatment, reverse transcription was performed following the instruction of SuperScript III Reverse Transcriptase (Invitrogen) using oligo (dT). Real-time reverse-transcription polymerase chain reaction (RT-PCR) was done using FastStart Universal SYBR Green Master (Roche). Primer sequences can be found in Supplementary Table 6

Bioinformatic and statistical analyses. Read processing and statistical methods were conducted following the criteria illustrated in Extended Data Fig. 4 Generally, Bowtie2 (ref. 33) was used to align reads to the Arabidopsis TAIR10 genome. Read assignment was achieved using HT-Seq ${ }^{34}$. Transcriptome and translatome changes were calculated using DESeq2 (ref. 35). Transcriptome fold changes $\left(\mathrm{RS}_{\mathrm{fc}}\right)$ for protein-coding genes were determined using reads assigned to exon by gene. Translatome fold changes $\left(\mathrm{RF}_{\mathrm{fc}}\right)$ for protein-coding genes were measured using reads assigned to CDS by gene. Translational efficiency was calculated by combining reads for all genes that passed reads per kilobase of transcript per million mapped reads (RPKM) $\geq 1$ in CDS threshold in two biological replicates and normalizing Ribo-seq RPKM to RNA-seq RPKM as reported ${ }^{12}$. The criteria used for uORF prediction are shown in Extended Data Fig. 6 and were performed using systemPipeR (https://github.com/tgirke/systemPipeR). The MEME online too ${ }^{36}$ was used to search strand-specific $5^{\prime}$ leader sequences for enriched consensuses compared with whole-genome $5^{\prime}$ leader sequences with default parameters. The density plot was presented using $\mathrm{IGB}^{37}$. The nucleotide resolution of the coverage around start and stop codons was performed using the 15th nucleotide of 30-nucleotide reads of Ribo-seq, similar as reported previously ${ }^{10,38}$. Whole-transcriptome R-motif search was performed using the FIMO tool in the MEME suite ${ }^{36}$. LUC/RLUC ratio was first tested for normal distribution using a Shapiro-Wilk test. A two-sided Student's $t$-test was used for comparison between two samples. Two-sided one-way or two-way analysis of variance was used for more than two samples, and Tukey's test was used for multiple comparisons. GraphPad Prism 6 was used for all the statistical analyses. Unless specifically stated, sample size $n$ means the biological replicate and experiment was performed three times with similar results. $* P<0.05$, $* * P<0.01$,
$* * * P<0.001$, and $* * * * P<0.0001$ indicate significant increases; NS, no significance; $\uparrow \dagger \uparrow P<0.001$ indicates a significant decrease.

Data availability. The authors declare that the main data supporting the findings of this study are available within the article and its Source Data files. Extra data are available from the corresponding author upon request. The RNA-seq and Ribo-seq data have been deposited in Gene Expression Omnibus under accession number GSE86581.

22. Nakagawa, T. et al. Development of series of gateway binary vectors, pGWBs, for realizing efficient construction of fusion genes for plant transformation. J. Biosci. Bioeng. 104, 34-41 (2007).

23. Curtis, M. D. \& Grossniklaus, U. A gateway cloning vector set for highthroughput functional analysis of genes in planta. Plant Physiol. 133, 462-469 (2003).

24. Xu, G. et al. One-step, zero-background ligation-independent cloning intron-containing hairpin RNA constructs for RNAi in plants. New Phytol. 187, 240-250 (2010).

25. Li, J. T. et al. Modification of vectors for functional genomic analysis in plants. Genet. Mol. Res. 13, 7815-7825 (2014).

26. Alonso, J. M. et al. Five components of the ethylene-response pathway identified in a screen for weak ethylene-insensitive mutants in Arabidopsis. Proc. Natl Acad. Sci. USA 100, 2992-2997 (2003)

27. Hua, J. et al. EIN4 and ERS2 are members of the putative ethylene receptor gene family in Arabidopsis. Plant Cell 10, 1321-1332 (1998).

28. Stepanova, A. N. Hoyt, J. M. Hamilton, A. A. \& Alonso, J. M. A link between ethylene and auxin uncovered by the characterization of two root-specific ethylene-insensitive mutants in Arabidopsis. Plant Cell 17, 2230-2242 (2005).

29. Galon, Y. et al. Calmodulin-binding transcription activator 1 mediates auxin signaling and responds to stresses in Arabidopsis. Planta 232, 165-178 (2010).

30. Clough, S. J. \& Bent, A. F. Floral dip: a simplified method for Agrobacteriummediated transformation of Arabidopsis thaliana. Plant J. 16, 735-743 (1998).

31. Ingolia, N. T., Brar, G. A., Rouskin, S., McGeachy, A. M. \& Weissman, J. S. The ribosome profiling strategy for monitoring translation in vivo by deep sequencing of ribosome-protected mRNA fragments. Nature Protocols 7 , 1534-1550 (2012).

32. Mustroph, A., Juntawong, P. \& Bailey-Serres, J. Isolation of plant polysomal mRNA by differential centrifugation and ribosome immunopurification methods. Methods Mol. Biol. 553, 109-126 (2009).

33. Langmead, B. \& Salzberg, S. L. Fast gapped-read alignment with Bowtie 2 . Nature Methods 9, 357-359 (2012)

34. Anders, S., Pyl, P. T. \& Huber, W. HTSeq-a Python framework to work with high-throughput sequencing data. Bioinformatics 31, 166-169 (2015).

35. Love, M. I., Huber, W. \& Anders, S. Moderated estimation of fold change and dispersion for RNA-seq data with DESeq2. Genome Biol. 15, 550 (2014).

36. Bailey, T. L. et al. MEME SUITE: tools for motif discovery and searching. Nucleic Acids Res. 37, W202-8 (2009)

37. Nicol, J. W., Helt, G. A., Blanchard, S. G., Jr, Raja, A. \& Loraine, A. E. The Integrated Genome Browser: free software for distribution and exploration of genome-scale datasets. Bioinformatics 25, 2730-2731 (2009).

38. Miettinen, T. P. \& Björklund, M. Modified ribosome profiling reveals high abundance of ribosome protected mRNA fragments derived from $3^{\prime}$ untranslated regions. Nucleic Acids Res. 43, 1019-1034 (2015). 
a

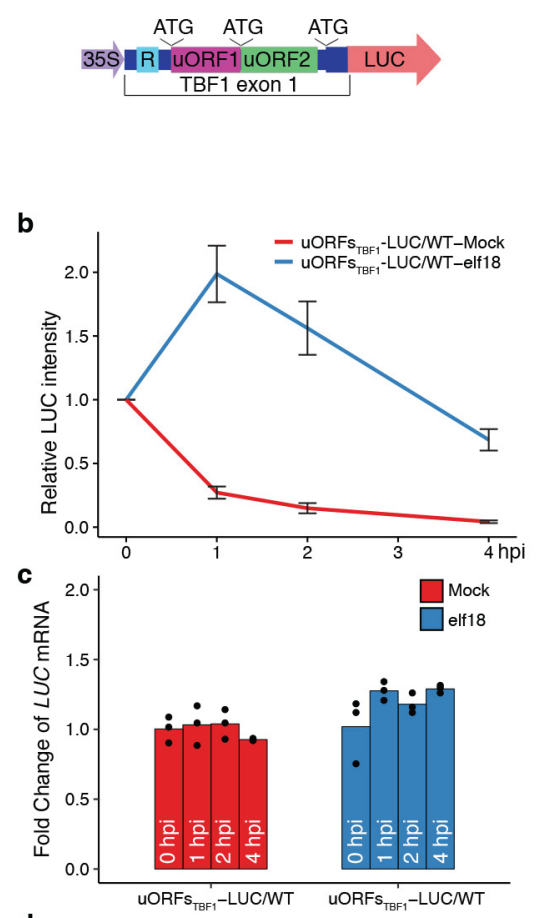

d
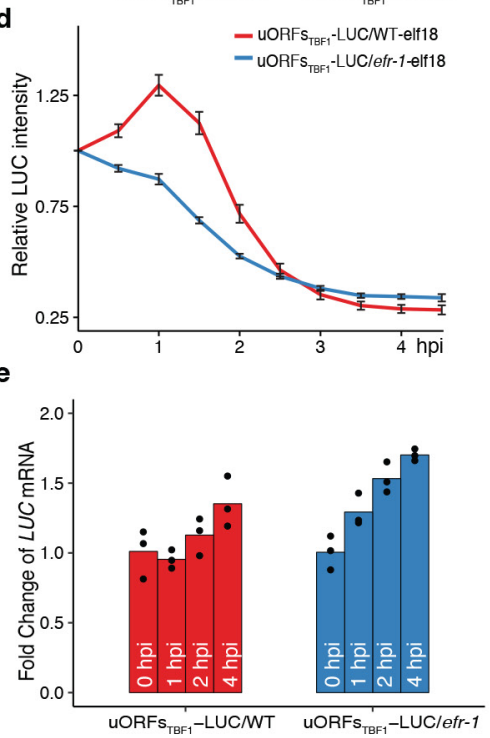

Extended Data Figure 1 | Translational activities during elf18-induced PTI. a, Schematic of the $35 S: u O R F s_{T B F 1}-L U C$ reporter, which is a fusion between the TBF1 exon 1 (uORF1/2 and sequence of the $\mathrm{N}$-terminal 73 amino acids) and the firefly luciferase gene (LUC) expressed constitutively by the CaMV 35S promoter. R, R-motif. b-e, Translation $(\mathbf{b}, \mathbf{d})$ and transcript levels (c, e) of the $35 \mathrm{~S}: u O R F s_{T B F 1}-L U C$ reporter in WT after Mock or elf18 treatment $(\mathbf{b}, \mathbf{c})$ or in WT and efr-1 upon elf18 treatment $(\mathbf{d}, \mathbf{e})$. LUC activity, mean \pm s.e.m. (b, $n=12 ; \mathbf{d}, n=9)$ after normalization to time 0 ; transcript levels, mean of fold changes normalized to time 0 with individual biological replicates shown as solid circles $(n=3)$; hpi, hours post infiltration. $\mathbf{f}-\mathbf{i}$, Polysome profiling of global translational activity $(\mathbf{f}, \mathbf{h})$ and $T B F 1$ mRNA translational activity calculated as ratios
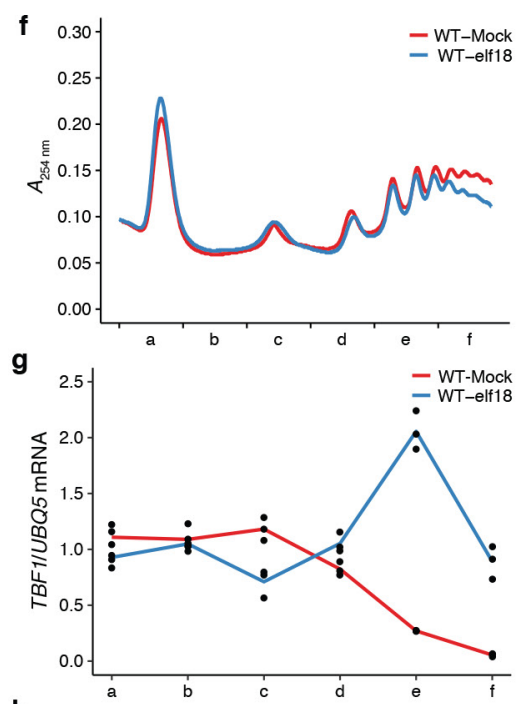

h

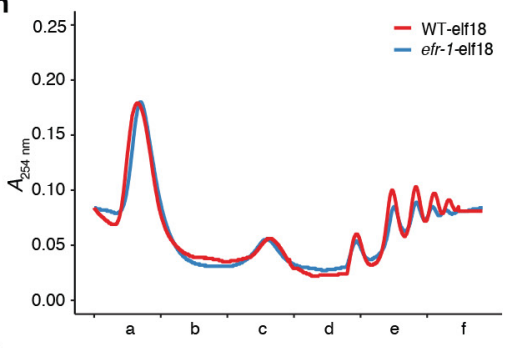

i

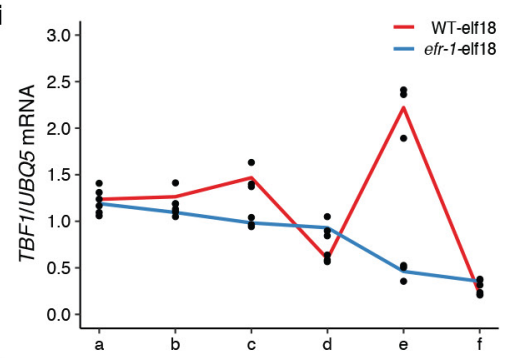

j

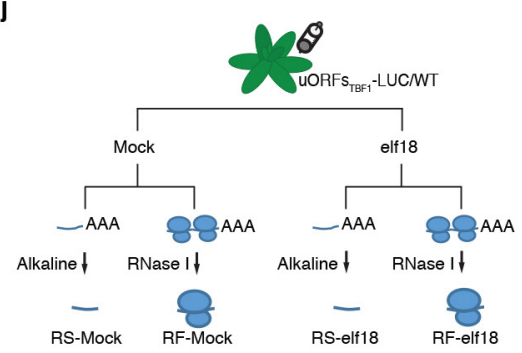

of polysomal/total mRNA (g, i) in WT after Mock or elf18 treatment (f, $\mathbf{g})$ or in WT and efr-1 upon elf18 treatment $(\mathbf{h}, \mathbf{i})$. Lower-case letters indicate polysomal fractions in polysome profiling indicated by sucrose gradient absorbance $\left(A_{254} \mathrm{~nm}\right)$. Expression levels of TBF1 were normalized against $U B Q 5$ level determined by RT-PCR in total mRNA and in polysomal fractions respectively. Data are shown as the relative TBF1 mRNA level in polysomal fractions after normalization to total TBF1 mRNA level. Bar with solid circles, mean with individual biological replicates $(n=3)$. j, Schematic of RNA-seq (RS) and Ribo-seq (RF) library construction using $u O R F s_{T B F 1}-L U C / \mathrm{WT}$ plants. RNase I and alkaline are two methods of generating RNA fragments. 


\section{RESEARCH LETTER}

a

RF \& RS fragment purification

\&

PNK treatment

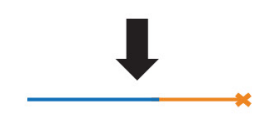

Purification of ligation products

Linker ligation

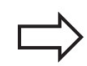

5'- $\mathrm{O}_{3} \mathrm{PO}$

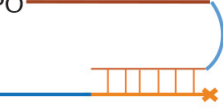

Reverse transcription
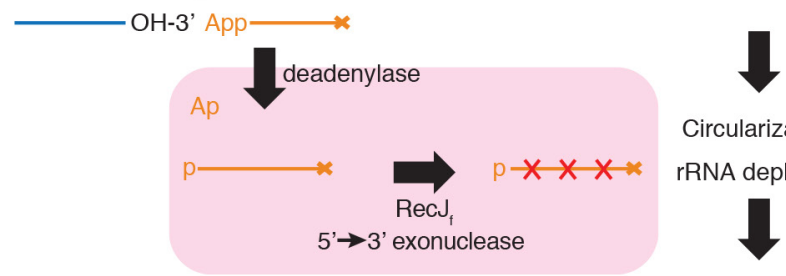

Circularization

rRNA depletion

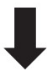

PCR amplification

and

barcode addition

b

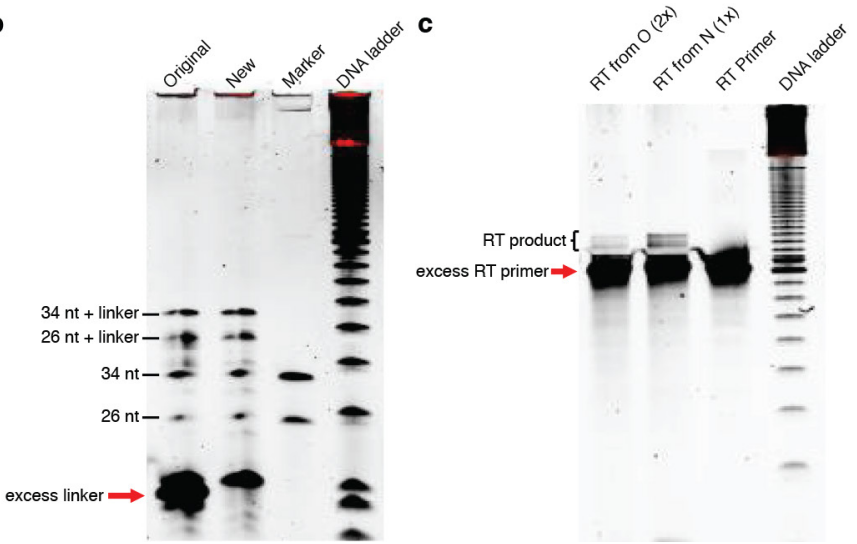

Extended Data Figure 2 Improvement made in the library construction protocol. a, Addition of $5^{\prime}$ deadenylase and $R e c J_{f}$ to remove excess

$5^{\prime}$ pre-adenylylated linker. mRNA fragments of RNA-seq and Ribo-seq were size-selected and dephosphorylated by PNK treatment, followed by $5^{\prime}$ pre-adenylylated linker ligation. The original method used gel purification to remove the excess linker. In the new method (pink background), $5^{\prime}$ deadenylase was used to remove pre-adenylylated group (Ap) from the unligated linker allowing cleavage by $\mathrm{Rec}_{\mathrm{f}}$. The resulting sample could then be used directly for reverse transcription. $\mathbf{b}$, The original (Original) and new (New) methods to remove excess linker were compared. Synthetic RNA markers of 26 and 34 nucleotides (nt) were used for linker ligation. RNA markers without the linker were used as controls. Arrow indicates the excess linkers. DNA ladder, 10 base pairs (bp). c, Reverse transcription (RT) showed the improvement of the new method over the original one. Half of the ligation mixture $(\mathrm{O})$ was gel purified to remove excess linkers before reverse transcription (loaded twice). The other half $(\mathrm{N})$ was treated with $5^{\prime}$ deadenylase and $\operatorname{Rec} \mathrm{f}_{\mathrm{f}}$, and directly used as template for reverse transcription (loaded once). Reverse transcription primers were loaded as control. Arrow indicates excess reverse transcription primers. 
a
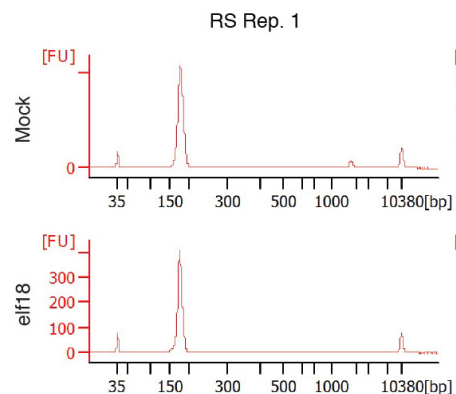

b

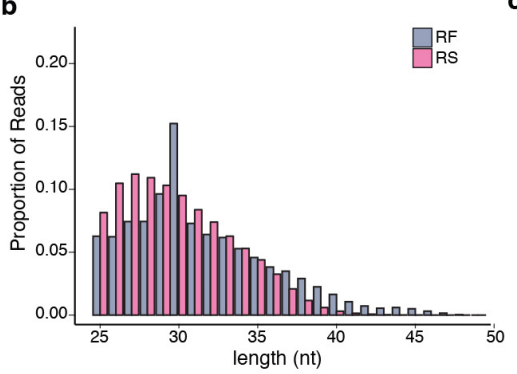

e
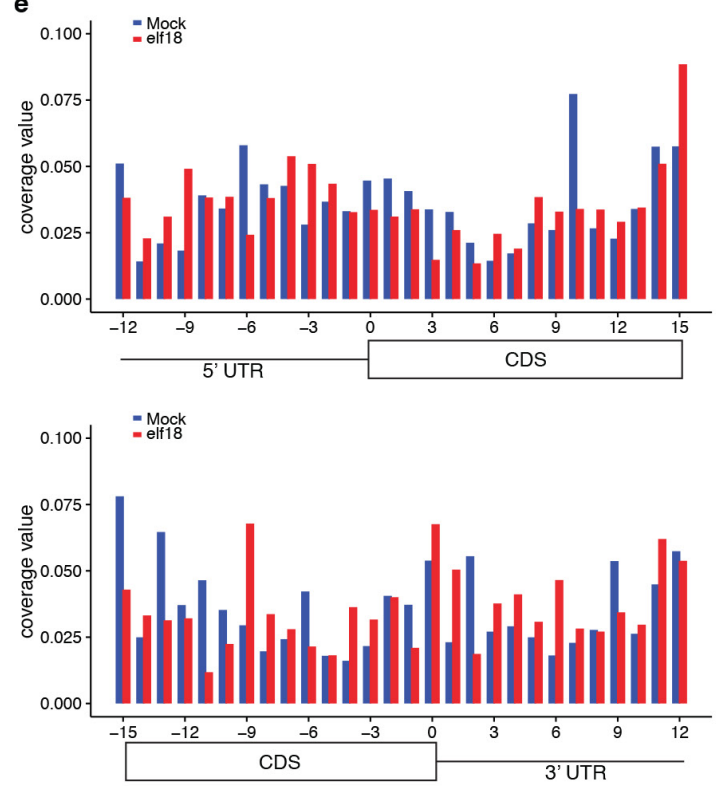

g

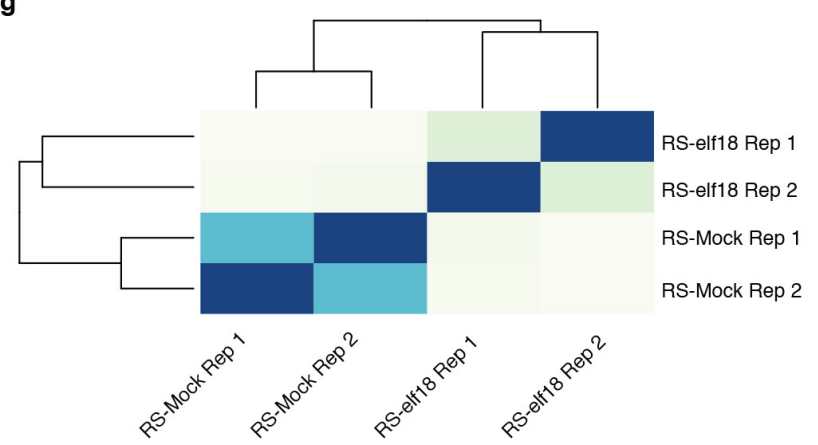

RS Rep. 2
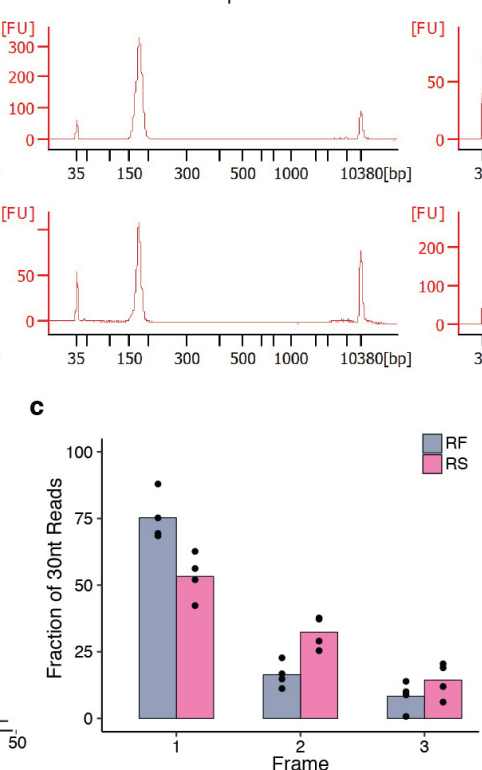

f
RF Rep. 1
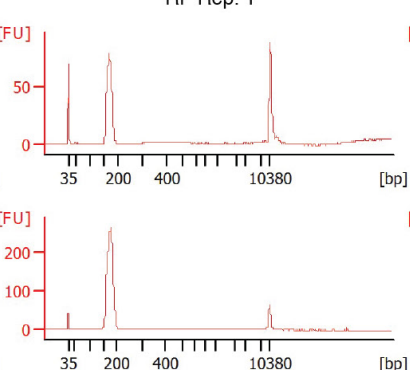

RF Rep. 2
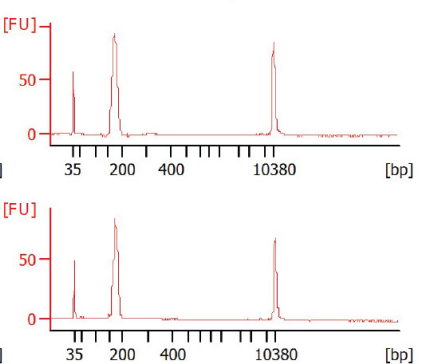

d $\square$ RS-Mock $\square$ RS-elf18 $\square$ RF-Mock $\square$ RF-elf18

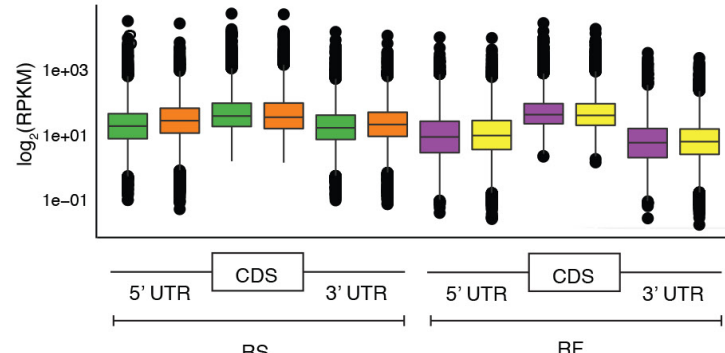

RS
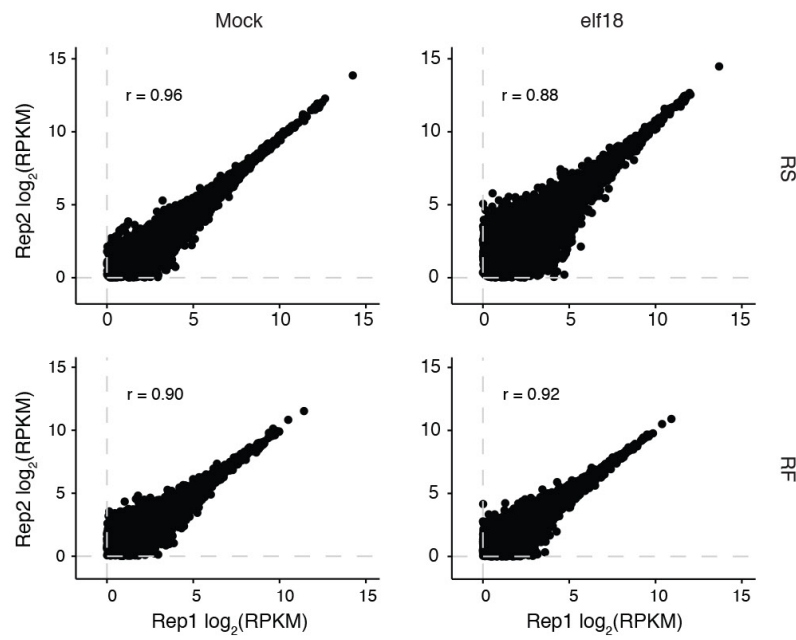

h

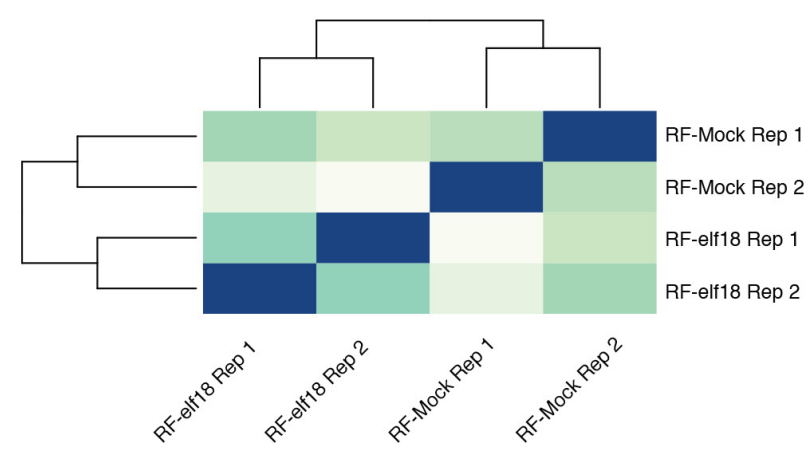

Extended Data Figure 3 | See next page for caption. 


\section{RESEARCH LETTER}

Extended Data Figure 3 Quality and reproducibility of RNA-seq and Ribo-seq libraries. Related to Fig. 1. a, BioAnalyzer profile showed high quality of RNA-seq (RS) and Ribo-seq (RF) libraries. In addition to internal standards ( $35 \mathrm{bp}$ and 10,380 bp), a single $\sim 170$ bp peak is present for RNA-seq and Ribo-seq libraries for Mock and elf18 treatments with both biological replicates (Rep1/2). b. Length distribution of total reads from four RNA-seq and four Ribo-seq libraries. c, Fraction of 30-nucleotide reads in total reads from four RNA-seq and four Ribo-seq libraries. Bar with solid circles, mean with individual biological replicates $(n=4)$ of percentage of reads with $5^{\prime}$ aligning to $\mathrm{A}$ (frame1),

$\mathrm{U}$ (frame2), and $\mathrm{G}$ (frame3) of the initiation codon. d, Read density along $5^{\prime}$ UTR, CDS, and 3' UTR of total reads from four RNA-seq and four

Ribo-seq libraries. Expressed genes with RPKM in CDS $\geq 1$ and length of UTR $\geq 1$ nucleotide were used for box plots. The top, middle, and bottom lines of the box indicate the 25th, 50th, and 75th percentiles, respectively. Filled circles represent RPKM values for individual outlier genes. e, Nucleotide resolution of the coverage around start and stop codons using the 15th nucleotide of 30-nucleotide reads of Ribo-seq. Reads in $3^{\prime}$ UTR may be due to digestion conditions that might favour the capture of ribosomes in different conformations associated with UTRs as previously observed ${ }^{10}$ and explained ${ }^{38}$. $\mathbf{f}$, Correlation between two replicates (Rep1/2) of RNA-seq and Ribo-seq samples. Data are shown as the correlation of $\log _{2}(\mathrm{RPKM})$ in CDS for expressed genes with RPKM in CDS $\geq 1$. Pearson correlation coefficient $r$ is shown. $\mathbf{g}, \mathbf{h}$, Hierarchical clustering showing the reproducibility between RNA-seq (g) and Riboseq (h) within two replicates (Rep1/2). Darker colour means greater correlation. 


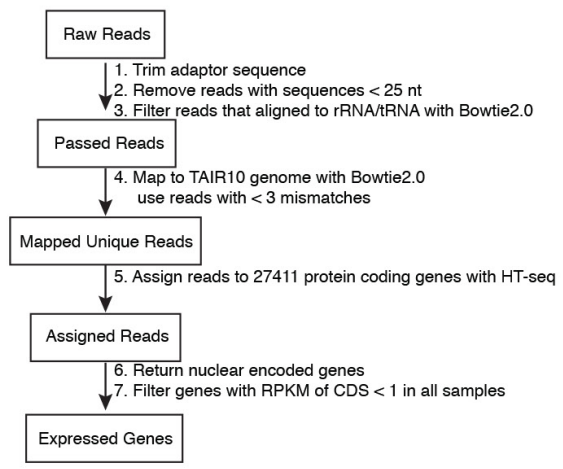

b

\begin{tabular}{llll|ll}
\hline & & RS-Mock & RS-elf18 & RF-Mock & RF-elf18 \\
\hline Raw number of reads & Rep1 & $47,065,199$ & $58,742,659$ & $133,768,593$ & $116,236,853$ \\
& Rep2 & $47,592,232$ & $58,270,271$ & $113,653,155$ & $125,304,695$ \\
\multirow{4}{*}{ Passed Reads } & Rep1 & $27,486,543$ & $26,884,242$ & $42,718,923$ & $51,033,470$ \\
& Rep2 & $18,843,216$ & $26,721,006$ & $51,905,987$ & $63,096,238$ \\
\multirow{4}{*}{ Unique mapped reads Rep1 } & $15,576,608$ & $11,988,097$ & $16,809,599$ & $24,748,709$ \\
& Rep2 & $8,463,878$ & $15,824,810$ & $24,866,878$ & $20,900,174$ \\
\hline
\end{tabular}

C

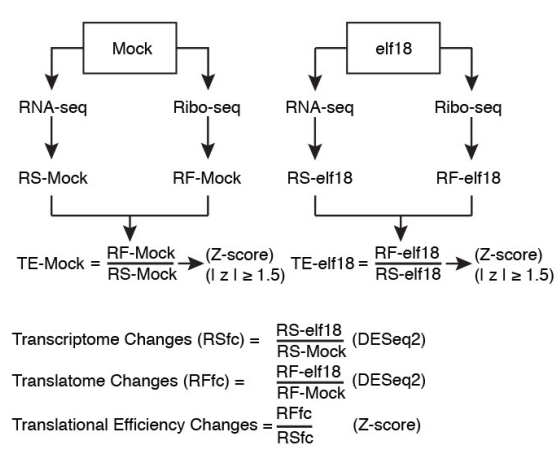

d

\begin{tabular}{lcc}
\hline \multicolumn{1}{c}{ GO Term } & Observed Frequency & $P$ value \\
\hline 1. GO:0010200 response to chitin & $7.80 \%$ & $9.80 \mathrm{E}-46$ \\
2. GO:0009743 response to carbohydrate stimulus & $8.40 \%$ & $2.02 \mathrm{E}-40$ \\
3. GO:0050896 response to stimulus & $31.50 \%$ & $8.69 \mathrm{E}-31$ \\
4. GO:0010033 response to organic substance & $14.40 \%$ & $1.57 \mathrm{E}-24$ \\
5. GO:0042221 response to chemical stimulus & $18.80 \%$ & $1.45 \mathrm{E}-22$ \\
6. GO:0006952 defense response & $10.50 \%$ & $1.77 \mathrm{E}-20$ \\
7. GO:0006950 response to stress & $18.90 \%$ & $9.80 \mathrm{E}-19$ \\
8. GO:0002376 immune system process & $5.80 \%$ & $2.73 \mathrm{E}-16$ \\
9. GO:0006955 immune response & $5.20 \%$ & $1.03 \mathrm{E}-14$ \\
10. GO:0051707 response to other organism & $7.70 \%$ & $1.42 \mathrm{E}-14$ \\
11. GO:0045087 innate immune response & $5.10 \%$ & $2.58 \mathrm{E}-14$ \\
12. GO:0051704 multi-organism process & $7.80 \%$ & $4.14 \mathrm{E}-14$ \\
13. GO:0009607 response to biotic stimulus & $7.90 \%$ & $5.72 \mathrm{E}-14$ \\
14. GO:0009620 response to fungus & $3.80 \%$ & $5.78 \mathrm{E}-12$ \\
\hline
\end{tabular}

Extended Data Figure 4 | Global analyses of transcriptome, translatome, and translational efficiency upon elf18 treatment. Related to Fig. 1. a, Flowchart for read processing and assignment. b, Reads after each processing. c, Statistical methods and criteria for transcriptome $\left(\mathrm{RS}_{\mathrm{fc}}\right)$, translatome $\left(\mathrm{RF}_{\mathrm{fc}}\right)$, and translational efficiency fold-change analyses. d, GO term enrichment analysis for RNA-seq upregulated genes. e, Normal distribution of $\log _{2}$ (translational efficiency) for Mock and elf18 treatment. $\mathbf{f}$, Translational efficiency changes in the endogenous

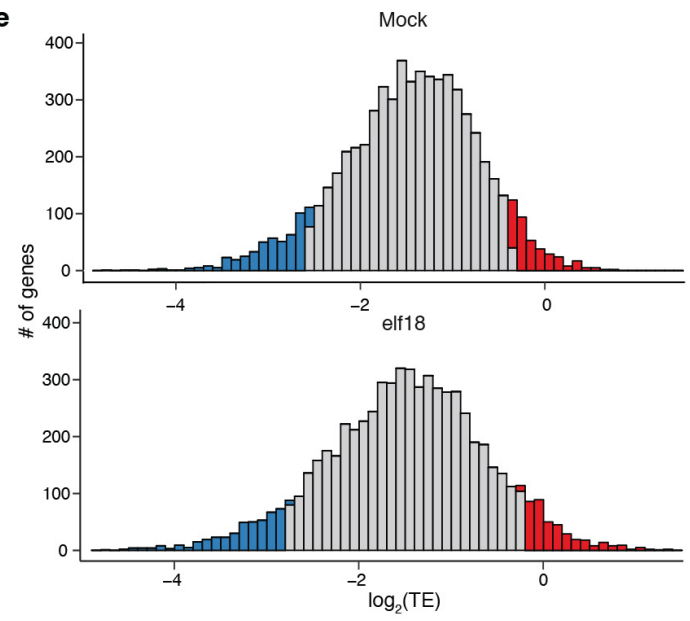

$f$
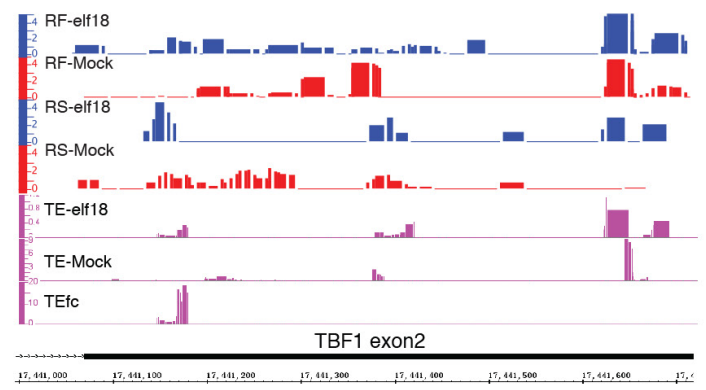

g

\begin{tabular}{lcc}
\hline \multicolumn{1}{c}{ GO Term } & Observed Frequency & Pvalue \\
\hline 1. GO:0050896 response to stimulus & $23.50 \%$ & $9.77 \mathrm{E}-04$ \\
2. GO:0006464 protein modification process & $10.60 \%$ & $3.43 \mathrm{E}-03$ \\
3. GO:0007166 cell surface receptor linked signal & $2.80 \%$ & $5.08 \mathrm{E}-03$ \\
4. GO:0009416 response to light stimulus & $5.30 \%$ & $5.08 \mathrm{E}-03$ \\
5. GO:0007165 signal transduction & $8.40 \%$ & $5.53 \mathrm{E}-03$ \\
6. GO:0006468 protein phosphorylation & $7.80 \%$ & $6.49 \mathrm{E}-03$ \\
7. GO:0016310 phosphorylation & $7.80 \%$ & $7.95 \mathrm{E}-03$ \\
8. GO:0016070 RNA metabolic process & $5.90 \%$ & $8.88 \mathrm{E}-03$ \\
\hline
\end{tabular}

h
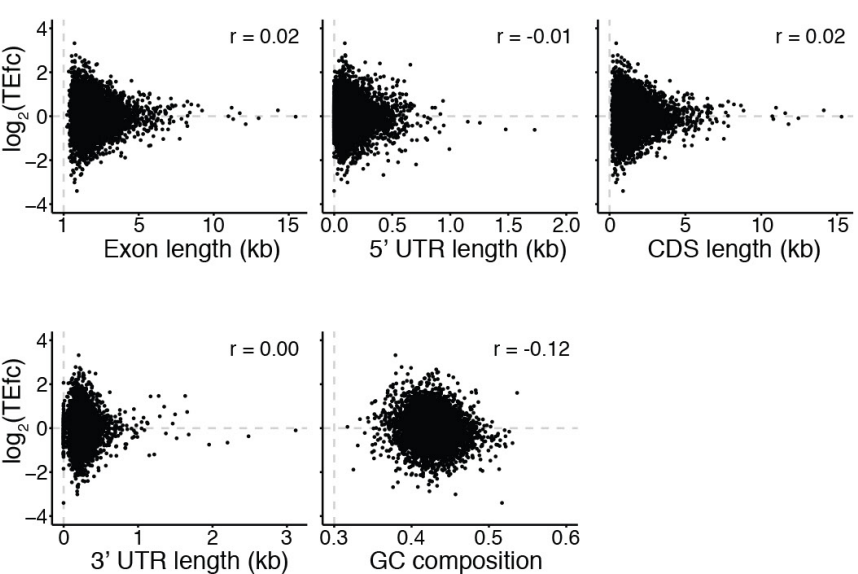

TBF1 gene. Read coverage was normalized to uniquely mapped reads with IGB. Translational efficiencies for the TBF1 exon 2 in Mock and elf18 treatments were determined to calculate translational efficiency fold change. $g$, GO term enrichment found in $\mathrm{TE}_{\text {up }}$ genes in response to elf18 treatment. A $z$ score $\geq 1.5$ was used. $\mathbf{h}$, Correlation between translational efficiency fold change and exon length, $5^{\prime}$ UTR length, $3^{\prime}$ UTR length, and guanine-cytosine (GC) composition. TE, translational efficiency. 
a
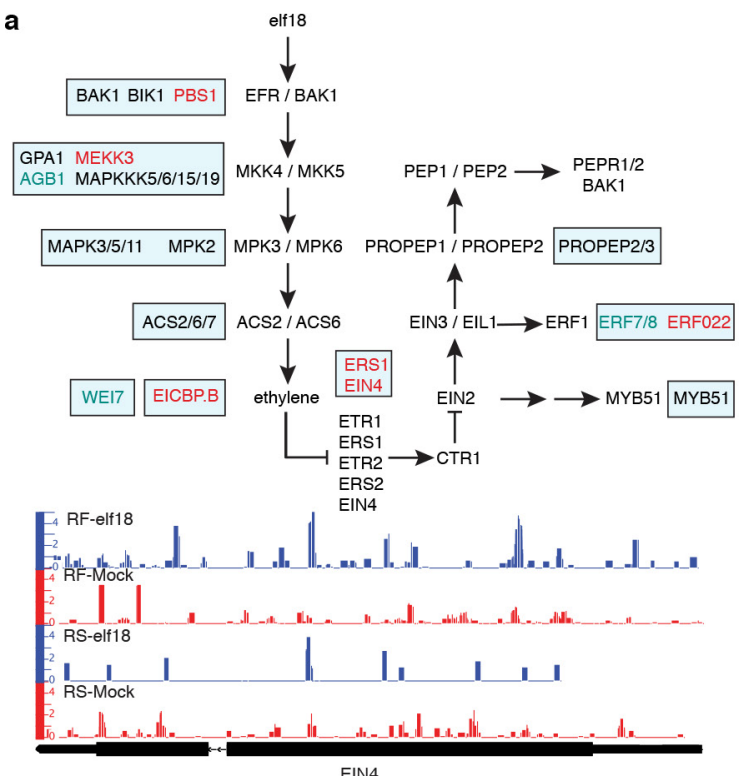

b

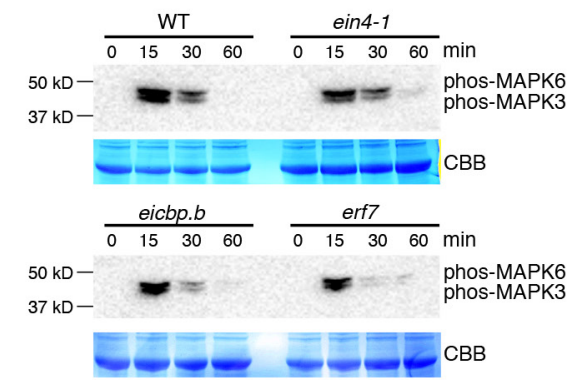

Extended Data Figure 5 | Characterization of novel PTI regulators, related to Fig. 1f. a, RNA-seq and translational efficiency changes in known or homologues of known components of the ethylene- and the damage-associated molecular pattern Pep-mediated PTI signalling pathways (top) and normalized distribution of RNA-seq and Ribo-seq reads of one example (that is, EIN4; bottom). The pathway was modified from ref. 13. In rectangular boxes: black, RNA-seq-changed; red, $\mathrm{TE}_{\text {up; }}$; blue, $\mathrm{TE}_{\mathrm{dn}}$. b, MAPK activation. Twelve-day-old ein4-1, eicbp.b, and erf7 seedlings were treated with $1 \mu \mathrm{M}$ elf18 solution and collected at the indicated time points for immunoblot analysis using the phosphospecific antibody against MAPK3 and MAPK6. See Supplementary Text for gel source data. c, Callose deposition. Three-week-old plants were infiltrated

e

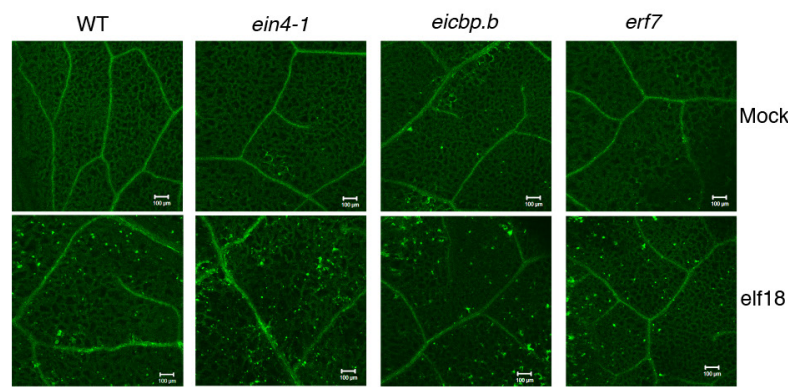

d
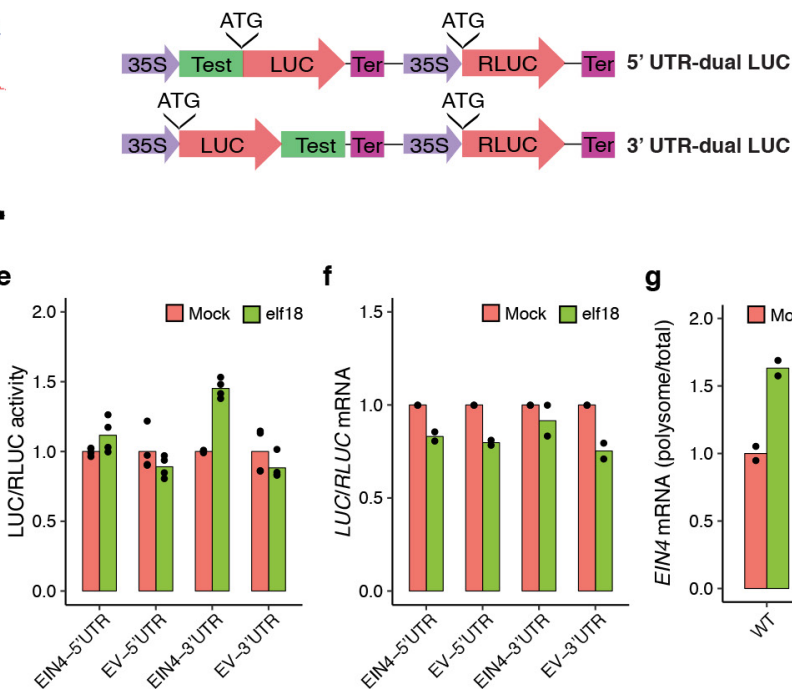

f
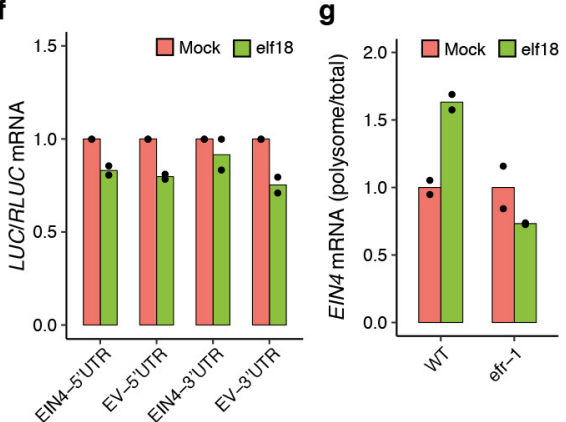

with $1 \mu \mathrm{M}$ elf18 or Mock. Leaves were stained $20 \mathrm{~h}$ later in aniline blue followed by confocal microscopy. Representative of five images. Scale bar, $100 \mu \mathrm{m}$. d, Schematic of the dual-LUC system. Test, $5^{\prime}$ leader sequence (including UTR) or $3^{\prime}$ UTR of the gene tested; LUC, firefly luciferase; RLUC, renilla luciferase, Ter, terminator. e, Dual-LUC assay of EIN4 UTRs on translational activity upon elf18 treatment in $N$. benthamiana $(n=4)$. f, Effects of EIN4 UTRs on ratios of $L U C / R L U C$ mRNA upon elf18 treatment (two experiments with three technical replicates). EV, empty vector. g, EIN4 translational activity upon elf18 treatment calculated as ratios of polysomal/total mRNA (two experiments with three technical replicates). Bar with solid circles, mean with individual biological replicates. 
a

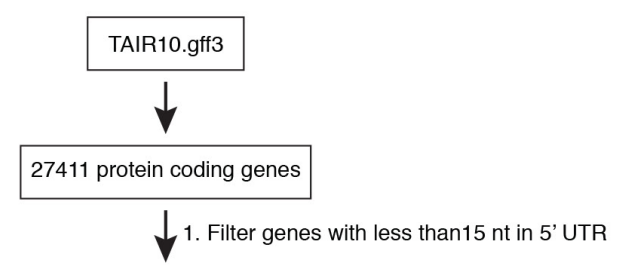

19412 5' UTR containing genes

Retrieve genomic sequences

TAIR10.fasta

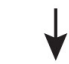

5' UTR.fasta

3. Predict UORF(>6 nt) with SystemPipe.R

6033 predicted uORF genes

- 9887 predicted uORFs

b

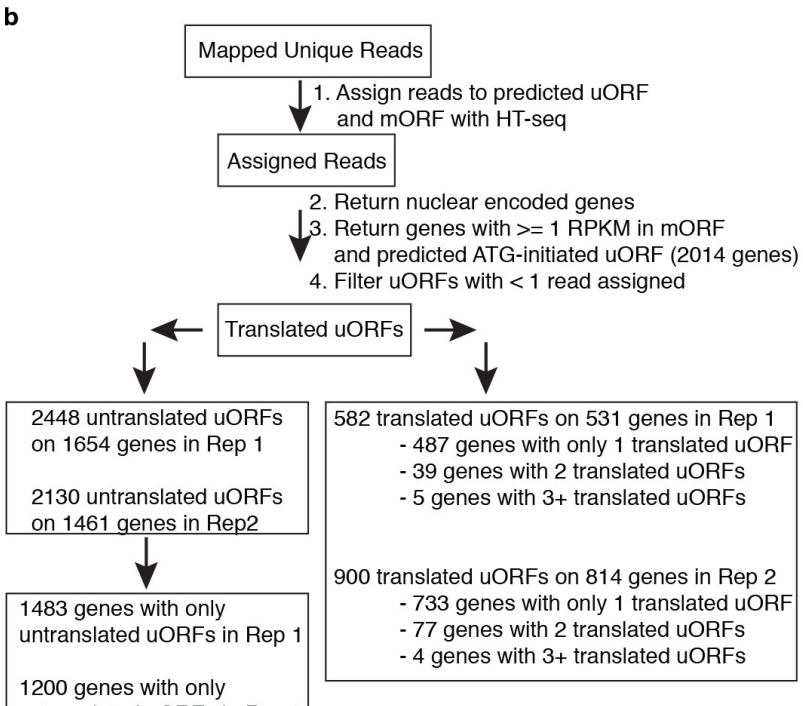

200 genes with only

untranslated uORFs in Rep 2

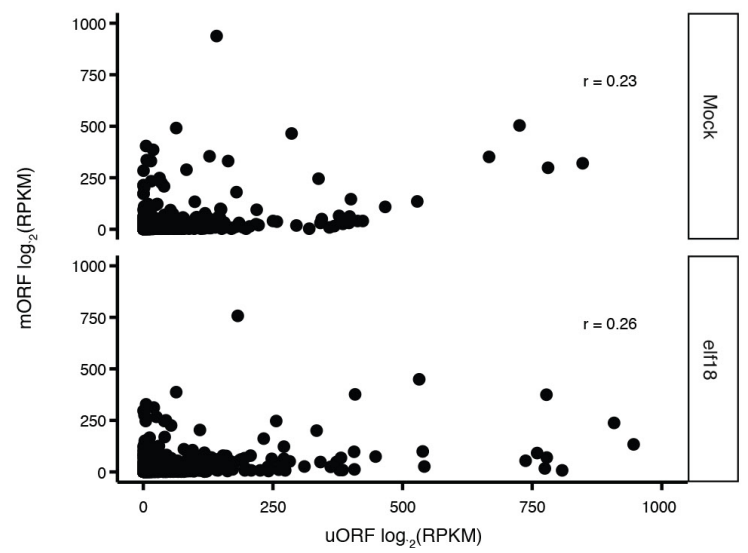

Extended Data Figure 6 | uORF-mediated translational control. a, b, Flowcharts of steps used to identify predicted (a) and translated (b) uORFs. c, Read density of uORF and mORF. For those genes with reads assigning to $\mathrm{uORF}$ and with $\mathrm{RPKM}$ in its $\mathrm{mORF} \geq 1, \log _{2}$ (RPKM) for individual uORFs and mORFs are plotted for Mock and elf18 treatment, respectively. $r$, Pearson correlation coefficient. d, Definition of mORF/uORF ratio shift between Mock and elf18 treatments. e, Histogram of $\mathrm{mORF} / \mathrm{uORF}$ shift upon elf18 treatment. The ratio of $\mathrm{mORF} / \mathrm{uORF}$ for elf18 divided by that for Mock was defined as the shift value. Data d

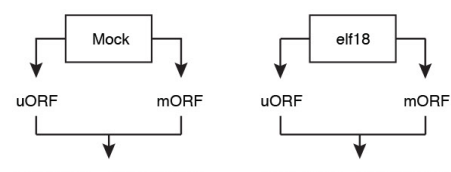

Ratio-Mock $=$ mORF/UORF $\quad$ Ratio-elf $18=$ mORF/UORF

Shift value $=\frac{\text { Ratio-elf } 18}{\text { Ratio-Mock }}(Z$-score $) ;|z| \geq 1.28$
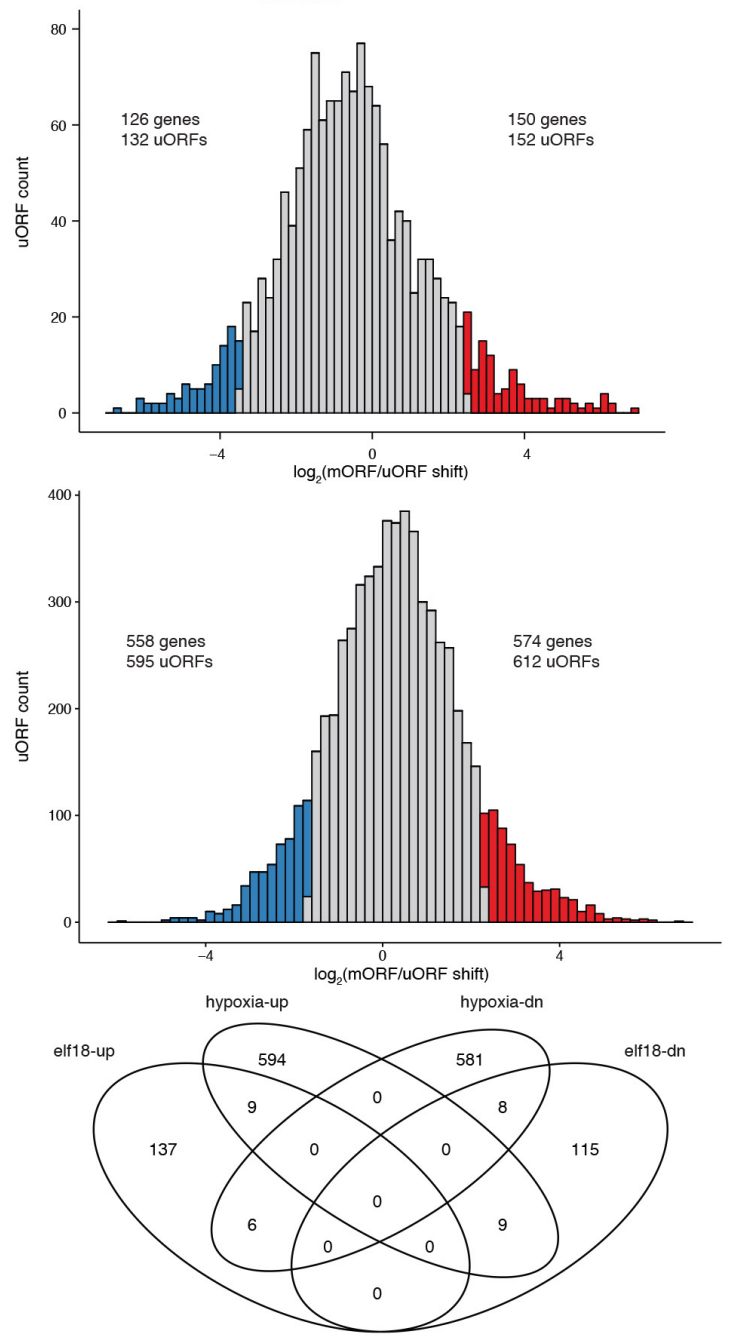

h

RF $\log _{2}$ (mORF/UORF)

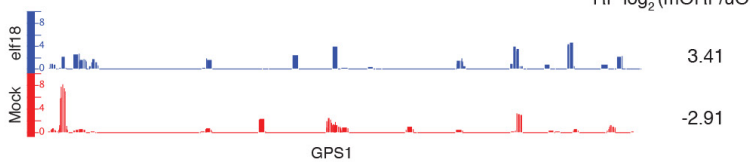

ab

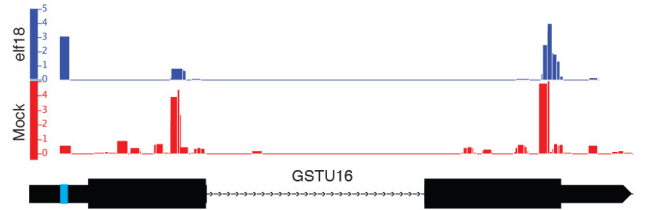

2.06

are shown as the distribution of the $\log _{2}$ transformation of shift values. uORFs with significant shift determined by $z$ score are coloured and whose numbers are shown. f, Histogram of mORF/uORF shift upon hypoxia stress ${ }^{10} \cdot \mathbf{g}$, Venn diagrams showing overlapping uORFs with significant ribo-shift in responses to elf18 and hypoxia treatments. h, Normalized distribution of RNA-seq and Ribo-seq reads to show ribo-shift of GPS1 (AT2G34630) and GSTU16 (AT1G59700) upon elf18 treatment. Numbers on the right mean $\log _{2}(\mathrm{mORF} / \mathrm{uORF})$ of Ribo-seq. uORFs are boxed with blue colour. 
a

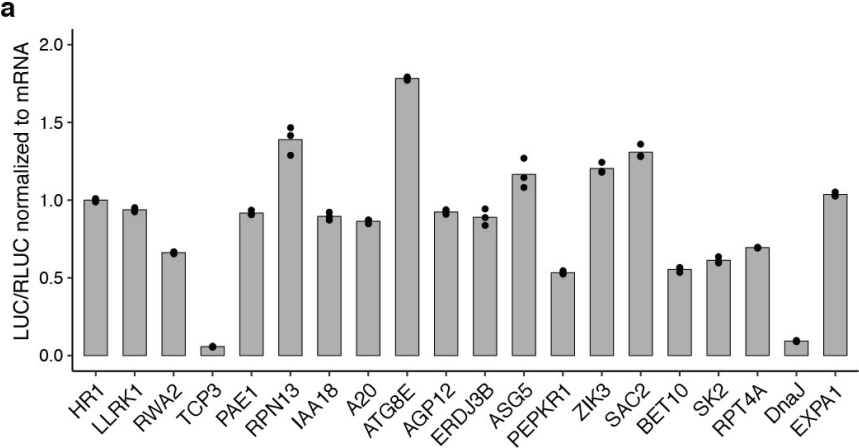

b

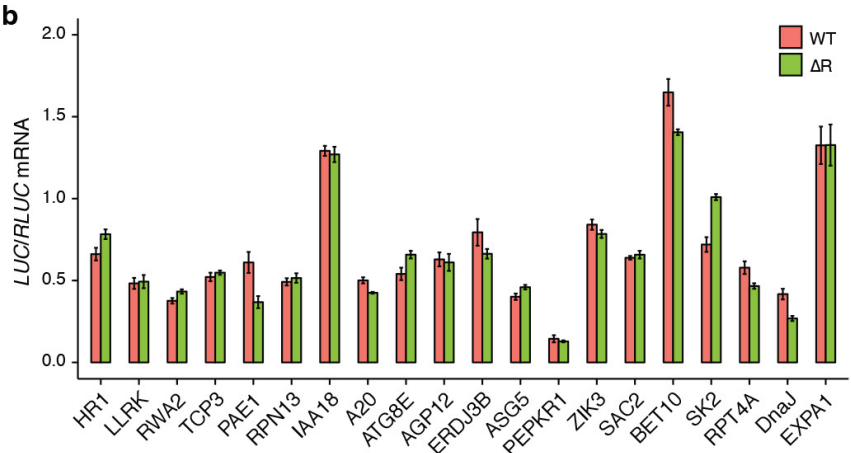

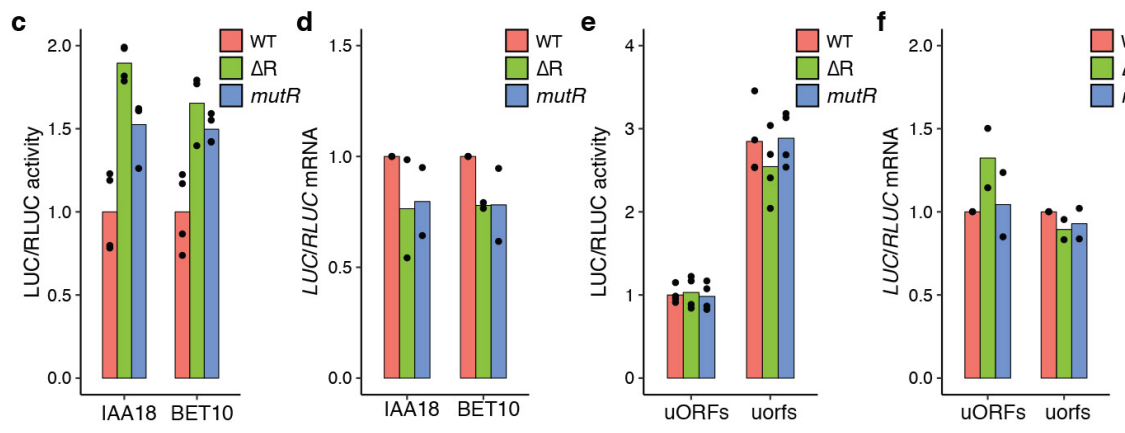

g

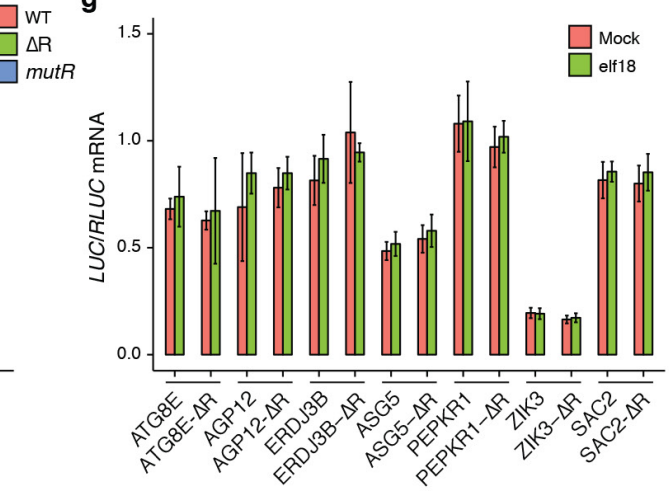

h

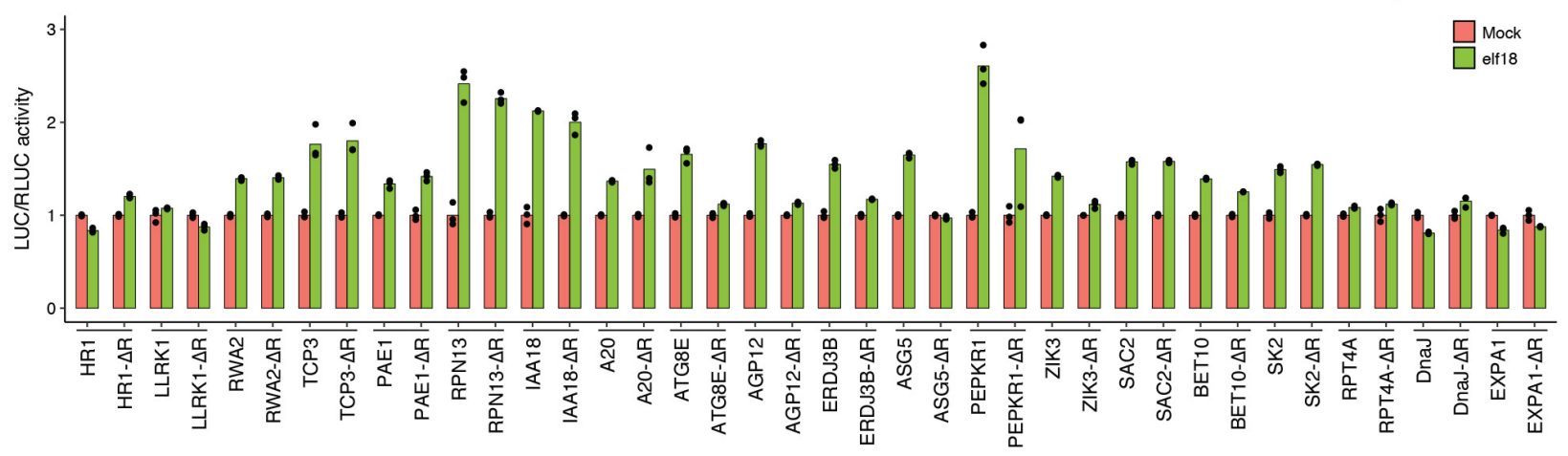

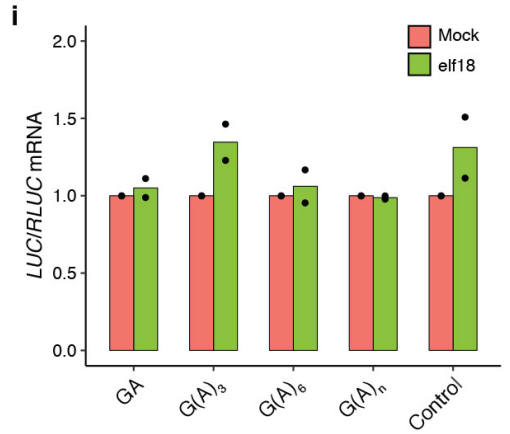

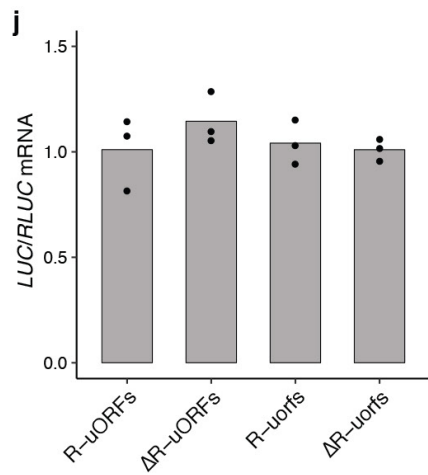

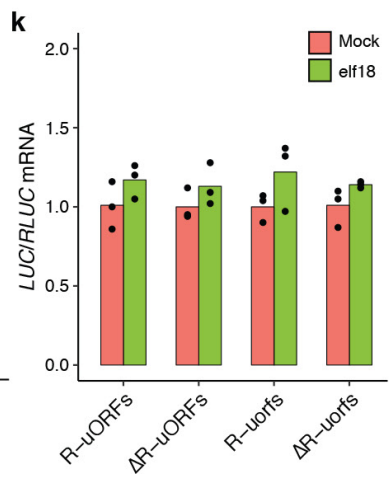

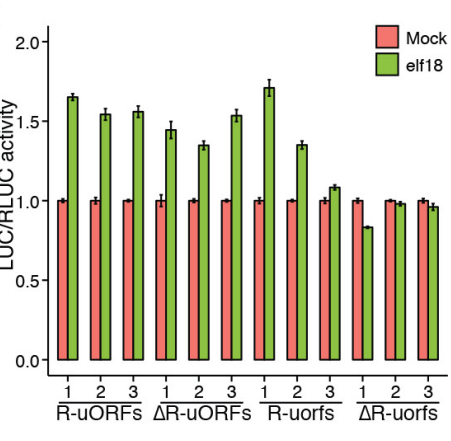

Extended Data Figure 7 | R-motif-mediated translational control in response elf18 induction. Related to Fig. 2. a, Effects of R-motif containing $5^{\prime}$ leader sequences on basal translational activities after normalization to mRNA $(n=3)$. $\mathbf{b}$, Effects of R-motif deletions $(\Delta \mathrm{R})$ on mRNA abundance $(n=6)$. $\mathbf{c}-\mathbf{f}$, Effects of R-motif deletion and R-motif substitution mutations on basal translation (c, e; $n=4)$ and mRNA levels (d, f, two experiments with three technical replicates) for IAA18 and BET10 (c, d) and TBF1 (e, f). g, mRNA levels in WT and R-motif deletion mutants with and without elf18 treatment $(n=9)$. h, Effects of R-motif deletions $(\triangle \mathrm{R})$ on translational responsiveness to elf18 measured using the dual-LUC assay $(n=3)$. i, Effects of $\mathrm{GA}, \mathrm{G}(\mathrm{A})_{3}, \mathrm{G}(\mathrm{A})_{6}$, and $\mathrm{G}(\mathrm{A})_{n}$ repeats on mRNA levels when inserted into $5^{\prime}$ UTR of the reporter in transient assay performed in $N$. benthamiana (two experiments with three technical replicates). $\mathbf{j}, \mathbf{k}$, Effects of R-motif deletion and/or uORF mutations on TBF1 mRNA abundance (j) and transcriptional responsiveness to Mock and elf18 treatments $(\mathbf{k}) ; n=3$ after normalization to WT $(\mathbf{j})$ or WT with Mock treatment (k). l, Contributions of R-motif and uORFs to TBF1 translational response to elf18 in transgenic Arabidopsis plants. Numbers 1,2 , and 3 on the $x$ axis represent individual transgenic lines tested $(n=6$ after normalization to Mock). Bar with solid circles, mean with individual biological replicates. 
a

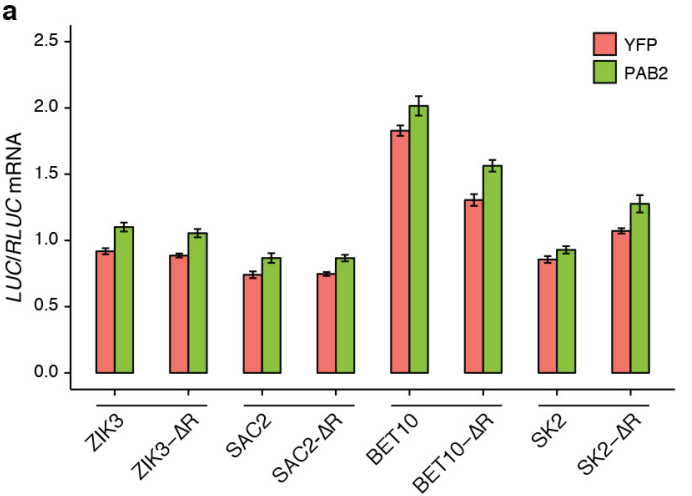

Extended Data Figure 8 Effects of PABs on mRNA transcription and PTI-associated phenotypes. Related to Fig. 3. a, Influence of coexpressing PAB2 on mRNA abundance $(n=9)$. $\mathbf{b}$, The elf18-induced seedling growth inhibition in WT, efr-1, pab2 pab4 (pab2/4), and pab2 b

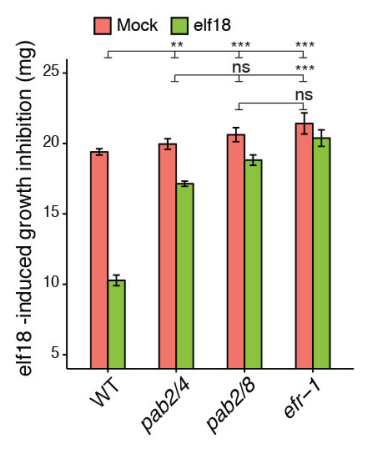

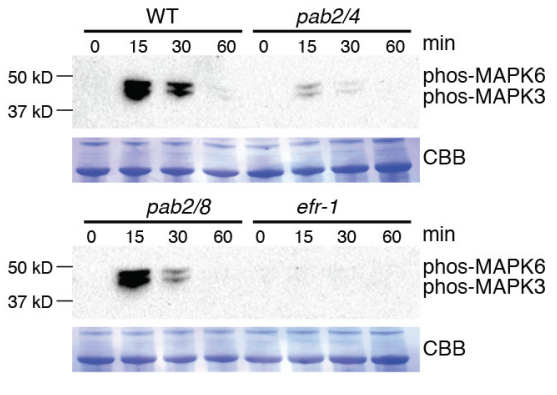

pab8 (pab2/8) (mean \pm s.e.m., $n=5)$. c, MAPK activation in WT, pab2/4, pab2/8, and efr-1 seedlings after elf18 treatment measured by immunoblotting using a phosphospecific antibody against MAPK3 and MAPK6. 


\section{RESEARCH LETTER}

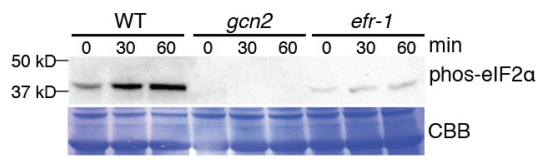

c

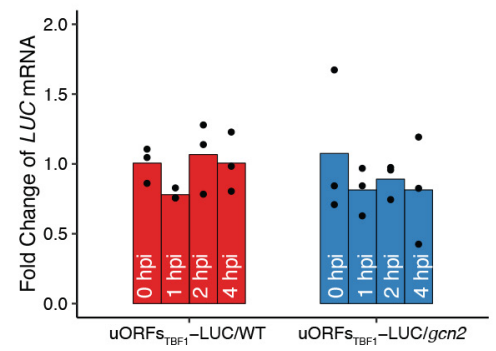

Extended Data Figure 9 | Roles of GCN2 in PTI in plants. a-d, Effects of the $g c n 2$ mutation on elf18-induced eIF2 $\alpha$ phosphorylation (a), translational induction (b, mean \pm s.e.m. of LUC activity, $n=8)$, and transcription of the $u O R F_{T B F 1}-L U C$ reporter $(\mathbf{c}, n=3$; bar with solid

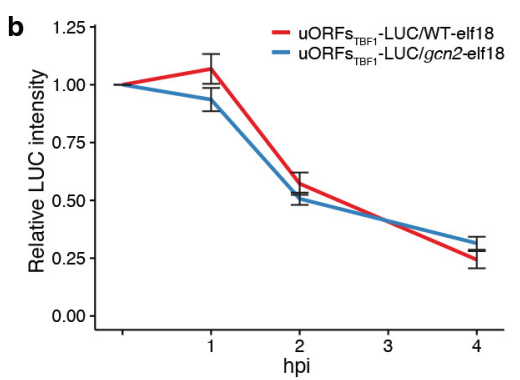

d

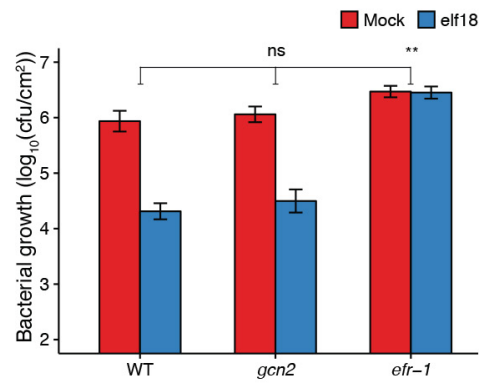

circles, mean with individual biological replicates), and resistance to Psm ES4326 (d, mean \pm s.e.m., $n=8$ ). See Supplementary Text for gel source data. 